"C 2019 IEEE. Personal use of this material is permitted. Permission from IEEE must be obtained for all other uses, in any current or future media, including reprinting/republishing this material for advertising or promotional purposes, creating new collective works, for resale or redistribution to servers or lists, or reuse of any copyrighted component of this work in other works." 


\title{
TOA-Based Passive Localization Constructed Over Factor Graphs: A Unified Framework
}

\author{
Weijie Yuan ${ }^{\circledR}$, Student Member, IEEE, Nan $\mathrm{Wu}^{\circledR}$, Member, IEEE, Qinghua Guo ${ }^{\circledR}$, Senior Member, IEEE, \\ Xiaojing Huang ${ }^{\circledR}$, Senior Member, IEEE, Yonghui $\mathrm{Li}^{\circledR}$, Fellow, IEEE, and Lajos Hanzo ${ }^{\circledR}$, Fellow, IEEE
}

\begin{abstract}
Passive localization based on time of arrival (TOA) measurements is investigated, where the transmitted signal is reflected by a passive target and then received at several distributed receivers. After collecting all measurements at receivers, we can determine the target location. The aim of this paper is to provide a unified factor graph-based framework for passive localization in wireless sensor networks based on TOA measurements. Relying on the linearization of range measurements, we construct a Forney-style factor graph model and conceive the corresponding Gaussian message passing algorithm to obtain the target location. It is shown that the factor graph can be readily modified for handling challenging scenarios such as uncertain receiver positions and link failures. Moreover, a distributed localization method based on consensus-aided operation is proposed for a large-scale resource constrained network operating without a fusion center. Furthermore, we derive the CramérRao bound (CRB) to evaluate the performance of the proposed algorithm. Our simulation results verify the efficiency of the proposed unified approach and of its distributed implementation.
\end{abstract}

Index Terms-Passive localization, time of arrival, wireless sensor networks, Forney-style factor graph, distributed processing, consensus operation, Cramér-Rao bound.

Manuscript received February 13, 2019; revised June 19, 2019; accepted July 17, 2019. Date of current version October 16, 2019. This work was supported by the "National Science Foun-dation of China (NSFC)" (Grant No.61471037, 61571041), "A Foundation for the Author of National Excellent Doctoral Dissertation of P. R. China (FANEDD)" (Grant No. 201445). L. Hanzo would like to acknowledge the financial support of the Engineering and Physical Sciences Research Council projects EP/Noo4558/1, EP/PO34284/1, COALESCE, of the Royal Society's Global Challenges Research Fund Grant as well as of the European Research Council's Advanced Fellow Grant QuantCom. This paper was presented in part at the 2018 IEEE VTC, Porto, Portugal. (Corresponding author: Nan Wu.)

W. Yuan was with the School of Information and Electronics, Beijing Institute of Technology, Beijing 100081, China. He is now with the School of Electrical Engineering and Telecommunications, University of New South Wales, Sydney, NSW 2052, Australia (e-mail: wjyuan@bit.edu.cn).

$\mathrm{N}$. Wu is with the School of Information and Electronics, Beijing Institute of Technology, Beijing 100081, China (e-mail: wunan@bit.edu.cn).

Q. Guo is with the School of Electrical, Computer and Telecommunications Engineering, The University of Wollongong, Wollongong, NSW 2522, Australia (e-mail: qguo@uow.edu.au).

$\mathrm{X}$. Huang is with the Faculty of Engineering and Information Technology, University of Technology Sydney, Ultimo, NSW 2007, Australia (e-mail: xiaojing.huang@uts.edu.au).

$\mathrm{Y}$. $\mathrm{Li}$ is with the Centre of Excellence in Telecommunications, School of Electrical and Information Engineering, University of Sydney, Sydney, NSW 2006, Australia (e-mail: yonghui.li@sydney.edu.au).

L. Hanzo is with the School of Electronics and Computer Science, University of Southampton, Southampton SO17 1BJ, U.K. (e-mail: 1h@ecs.soton.ac.uk).

\section{INTRODUCTION}

$\mathbf{O}$ VER the last decades, the advances in low cost sensors have enabled the development of compelling applications in communications [1]. The sensor nodes which can communicate with each other through wireless links have been deployed for environmental monitoring, temperature detection, emergency and rescue operations as well as for many other applications of wireless sensor networks (WSN) [2], [3].

In general, the nodes are located based on so-called anchor nodes with known positions and their related inter-node measurements using radio frequency signals. A variety of measurement techniques have been considered for WSN-aided localization, which can be broadly divided into angle of arrival (AOA) [4], time of arrival (TOA) [5] and received signal strength (RSS) [6] based techniques. The disadvantages of AOA-based methods are that their specific required array structures are unsuitable in some scenarios and that their AOA measurements are sensitive to the multipath components. By contrast, the RSS-based method are often more attractive, since they are based on standard features of the sensors and they do not impose substantial power consumption. However, the performance of RSS-based methods also remains limited by the fading of wireless channels. Finally, the TOA-based methods measure the time required for the signal to travel from the transmitter to a receiver. Using ultra-wideband (UWB) signaling in TOA measurements is capable of providing highprecision range measurement [7], [8]. In this contribution, we mainly focus our attention on the TOA-based method.

The general family of localization problems can be further classified as active and passive localization [9], as shown in Fig. 1. In contrast to conventional active localization, the nodes to be located in passive scenario do not have the ability to obtain range measurements. To determine the location of passive target, a transmitter sends a signal which is reflected or relayed by the passive target, which is then captured by the receivers. Most of the TOA based localization techniques fall into the category of active localization [10]. For passive localization, a two-step estimation (TSE) method was proposed in [11] based on TOA measurements. However, Shen et al. [11] assume having synchronous transceivers with accurately known positions, which may be unrealistic in practical applications. In this work, we aim for addressing the main challenges in TOA based passive localization, which can be summarized as follows: 


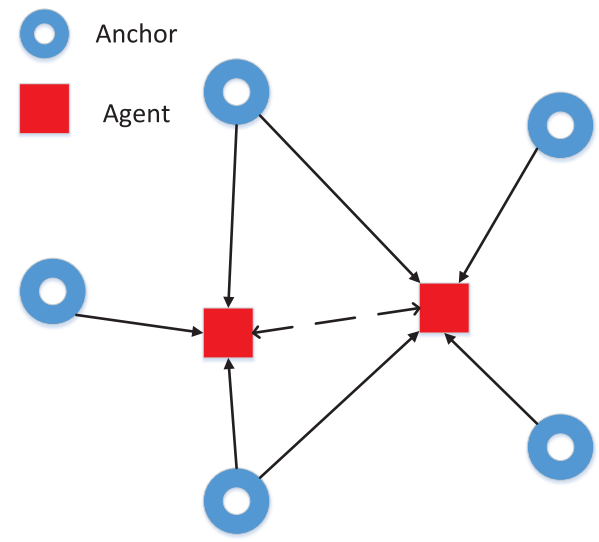

(a) Active localization case.
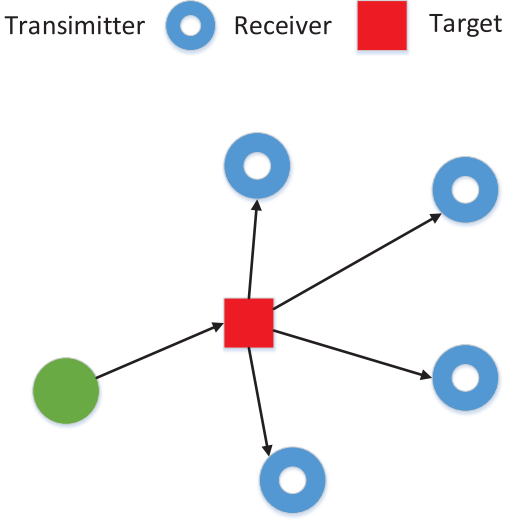

(b) Passive localization case.

Fig. 1. Active and passive localization case. In active case, the target (agent) has the ability of locating itself based on the measurements from sensors with known positions (anchor). In passive case, the measurements are obtained at receivers by acquiring the signals from the transmitter and reflected by the target.

1. Inaccurate positions of transceivers: In WSN, the size and cost limit of sensor nodes result in constrained resources, such as energy, memory and communications bandwidth. Equipping the transmitter and all receivers with a location device such as global positioning system (GPS) may lead to an excessive cost and energy consumption. The resultant erroneous position information will severely degrade the overall system performance. The problem of localization in the face of anchor position uncertainty has been extensively investigated in active localization [12], [13]. To elaborate, the target location can be accurately determined in the presence of inaccurate anchor positions based on the method of moments [12] and Bayesian filtering [13]. In our prior work [14], we have studied the passive localization of targets in the face of inaccurate receiver positions based on belief propagation. However, the target was assumed to be static and the impact of the transmitter's uncertain position was not investigated in detail.

2. Time synchronization: In TOA based measurement methods, having accurate clock synchronization is a vital requirement. Even a slight clock bias will lead to significant performance loss. ${ }^{1}$ Generally, network synchronization can be achieved by packet-based synchronization before localization [15]. However, in harsh environments, the oscillator is affected and the clock bias varies with time. Consequently, the network has to be frequently re-synchronized, which imposes high power consumption. Taking the close relationship between synchronization and localization into account, sophisticated schemes have been proposed for simultaneous synchronization and active localization [16]-[18]. In passive localization, Zhou et al. [19] exploited the time difference of arrival (TDOA) to eliminate impact of imperfect clock.In [20], a maximum likelihood method relying on Tinkhonov regularization was proposed for quasi-synchronous networks, while an expectation maximization based method was devised in [21], which treated the clock offsets of the receivers as hidden variables. In these treatises, the clock information is time invariant and the inherent clock skew is not considered.

3. Outliers in WSN: Localization in WSNs is based on measurements collected from multiple sensors. The measurements may be prone to unexpected changes in harsh environmental conditions. Furthermore, if there exist malicious sensor nodes in a WSN, flawed measurements will occur. We term the flawed observations which differ significantly from the normal readings as outliers. These outliers have to be detected in order to maintain high localization accuracy. A survey of outlier detection methods was provided in [22]. An efficient RSS measurements based approach was proposed in [23] for indoor environments. Jian et al. [24] defined the outlier detection problem of network localization and laid the theoretical foundations of outlier identification.

On the other hand, in the existing passive localization methods, a fusion center is required and the estimation process is executed centrally. However, in energy-constrained WSNs, the prohibitively high cost of transmitting information to a distant fusion center is undesirable. Hence conceiving effective distributed processing algorithms for WSNs is desirable. By exchanging local information with single-hop neighbor sensors, the distributed algorithm is able to estimate the unknown variables almost as accurately as if the measurements of other sensors were made explicitly available. To achieve this, the range measurements can be directly shared within the WSN, which regards each sensor as a fusion center. Based on the idea of consensus proposed by Tsitsiklis [25], likelihood consensus has been proposed by Meyer et al. in [26] for sensor self-localization and distributed tracking. In likelihood consensus (LC), the global likelihood function (GLF) is constructed as the product of local likelihood functions (LLF). The GLF can be obtained at each sensor by exchanging LLFs amongst each other. As a result, all sensors reach consensus on the GLF. To tackle the problem caused by nonlinear terms in LLF, distributed particle filtering (DPF) was proposed by Hlinka et al. in [27]. DPF is effective, but it suffers from high communication overhead and complexity, which hinders its implementation in WSNs.

\footnotetext{
${ }^{1}$ The impact of slight clock bias on communication links can be neglected since the synchronization constraint for communication is at the order of micro seconds.
} 
In this contribution, we first represent the conventional passive localization problem of [11] by a factor graph [28]. ${ }^{2}$ Then the classic message passing algorithm is invoked for determining the a posteriori distribution of target location based on the factor graph. However, the integration to be carried out in message updating is intractable, since the Euclidean norm involved in range measurements is nonlinear. Particle filtering (PF) [29] based methods are capable of solving this problem but the associated complexity is very high. To address this problem, the nonlinear Euclidean term in range measurements is linearized based on Taylor expansion. Consequently, we arrive at a linear model, which can be represented by a Forney-style factor graph and then Gaussian message passing [30] can be employed. Then we extend the factor graph framework to address the aforementioned three main challenges faced in TOA based passive localization. It is shown that the factor graph corresponding to different problems can be readily modified by adding corresponding factor nodes and edges. Additionally, considering a WSN operating without a fusion center, a distributed implementation of the proposed factor graph approach is proposed. Motivated by the fact that the 'belief' of a variable is the product of all incoming messages determining this variable, and that the means as well as covariances of messages can be calculated locally at each receiver, we can rely on the consensus method to reach agreement concerning the mean and the covariance matrix. Since only the vectors of mean values and covariance matrices are exchanged among the network nodes, the cost is much lower than that of DPF.

Briefly, the main contributions of this paper are summarized as follows,

- The problem of jointly locating moving passive targets in the face of imperfect transceivers is considered. A Forney-style factor graph representation [28] is conceived for low-complexity Gaussian message passing algorithm.

- The passive localization problem of a time-variant asynchronous network is solved. By approximating the range measurements related to the TOA information and target location, a state space model is constructed and the GMP algorithm [31] is implemented based on the corresponding factor graph.

- Based on the factor graph framework, a GMP algorithm is conceived for detecting link failures and simultaneously for passive localization. Furthermore, to tackle the calculation of messages at a product node, a variational message passing algorithm is developed.

- Distributed methods based on the consensus concept is proposed for distributively determining the parameters at each local receiver.

- The Cramér-Rao bound is derived to evaluate the performance of the proposed algorithms.

The rest of this paper is organized as follows. In Section II, the conventional passive localization model is introduced. Then in Section III we develop our factor graph based

\footnotetext{
${ }^{2}$ Localization based on the factor graph framework has already been investigated in the literature, e.g., [10], [14], [16], [18], [27], but mostly in the area of active localization.
}

approach to address the challenges of passive localization. Section IV presents our distributed processing algorithm based on the consensus method. We then derive the CramérRao Lower Bound for passive localization in Section V. In Section VI, our simulation results are provided and discussed. Finally, our conclusions are offered in Section VII.

Notation: $(\cdot)^{T}$ and $(\cdot)^{-1}$ denote the transpose and the inverse operator, respectively; $\|\cdot\|$ denotes the Euclidean norm; $\hat{\imath}$ denotes the estimate of an unknown variable; $\mathbb{E}$ denotes the expectation operator; $\delta(\cdot)$ denotes the Dirac delta function; $\mathcal{N}\left(x, m_{x}, V_{x}\right)$ denotes a Gaussian distribution of variable $x$ with mean $m_{x}$ and variance $V_{x}, \mathcal{N}\left(\mathbf{x}, \mathbf{m}_{\mathbf{x}}, \mathbf{V}_{\mathbf{x}}\right)$ denotes a Gaussian distribution of vector variable $\mathrm{x}$ with mean vector $\mathbf{m}_{\mathbf{x}}$ and covariance matrix $\mathbf{V}_{\mathbf{x}} ; \propto$ denotes the function on the left hand is proportion to the right hand; the $\nabla_{x}$ denotes the differential operator with respect to $x ; \mathbf{1}_{M}$ denotes an $M$-dimensional row vector with all components being 1; $\operatorname{diag}\{\mathbf{x}\}$ denotes a diagonal matrix with the main diagonal entries being the elements of $\mathrm{x}$ and the entries outside the main diagonal are all zero; covx denotes the covariance matrix of $\mathbf{x} ; \mathbf{A} \succeq \mathbf{B}$ denotes that $\boldsymbol{A}-\boldsymbol{B}$ is positive semi-definite.

\section{PRELIMINARIES}

As shown in Fig. 1(b), we consider a WSN constitutes of one target, one transmitter and $M=|\mathcal{M}|$ receivers, where $\mathcal{M}$ is the set of all receivers. ${ }^{3}$ The target moves on the plane while the transmitter and the receivers are static. Without loss of generality, we denote the location of target at time instant $k$ by $\mathbf{x}^{k}=\left[x^{k}, y^{k}\right]^{\mathrm{T}}$, the location of transmitter by $\mathbf{x}_{t}=\left[x_{t}, y_{t}\right]^{\mathrm{T}}$, the location of the $i$ th receiver by $\mathbf{x}_{i}=\left[x_{i}, y_{i}\right]^{\mathrm{T}}$ and $\mathbf{x}_{r}=\left[\mathbf{x}_{1}^{\mathrm{T}}, \ldots \mathbf{x}_{M}^{\mathrm{T}}\right]^{\mathrm{T}}$ as the collection of all receivers' positions. An impulse sent by the transmitter is reflected by the target and then received by $M$ receivers. ${ }^{4}$ For the $i$ th receiver, the observed signal propagation time $t_{i}^{k}$ from the transmitter to the $i$ th receiver via the target can be determined from TOA measurements as 5

$$
t_{i}^{k}=\left(\left\|\mathbf{x}^{k}-\mathbf{x}_{t}\right\|+\left\|\mathbf{x}^{k}-\mathbf{x}_{i}\right\|\right) / c+\omega_{i}^{k},
$$

with $c$ and $\omega_{i}^{k}$ denoting the speed of light and the measurement noise. Multiplying both sides of (1) by $c$, the range measurement from the transmitter via the target to the $i$ th receiver follows

$$
z_{i}^{k}=d_{i}^{k}+w_{i}^{k}=\left\|\mathbf{x}^{k}-\mathbf{x}_{t}\right\|+\left\|\mathbf{x}^{k}-\mathbf{x}_{i}\right\|+w_{i}^{k} .
$$

The range measurement noise $w_{i}^{k}$ is modeled as a zero mean Gaussian variable with variance $\left(\sigma_{i}^{k}\right)^{2}$. We assume that the

\footnotetext{
${ }^{3}$ The single target model can be extended to a multiple targets one, in which the separation of targets is important. Several methods, e.g. blind source separation and data association have been developed for this purpose, see [32], [33] for details.

${ }^{4}$ The multipath effect and clutters will affect the localization performance. We can employ background subtraction method, such as RIMAX algorithm [34] to extract the TOA measurements to receivers.

${ }^{5}$ The TOA measurement will be affected by imperfect observation conditions, such as measurement noise, variations of clock, baffle objects and sensor failures. For clarity of exposition, herein we only consider the conventional passive localization [11] with TOA measurement noise. Other environmental impairments are modeled and discussed in the following sections, respectively.
} 
noise at different time instants is independent and identically distributed, i.e., $\left(\sigma_{i}^{k}\right)^{2}=\sigma_{i}^{2}, \forall k$. Thus the likelihood function is given by

$p\left(z_{i}^{k} \mid \mathbf{x}^{k}\right)=\frac{1}{\sqrt{2 \pi \sigma_{i}^{2}}} \exp \left(-\frac{\left(z_{i}^{k}-\left\|\mathbf{x}^{k}-\mathbf{x}_{t}\right\|-\left\|\mathbf{x}^{k}-\mathbf{x}_{i}\right\|\right)^{2}}{2 \sigma_{i}^{2}}\right)$,

Furthermore, we denote the collection of all range measurements at instant $k$ as $\mathbf{z}^{k}=\left[z_{1}^{k}, \ldots, z_{M}^{k}\right]^{\mathrm{T}}$ and $\mathbf{z}=$ $\left[\left(\mathbf{z}^{1}\right), \ldots, \mathbf{z}^{n}\right]$, and the collection of target locations as $\mathbf{x}=$ $\left[\mathbf{x}^{1}, \ldots, \mathbf{x}^{n}\right]$. Since the noise samples at different time instants and receivers are independent, we formulate the global likelihood function as,

$$
p(\mathbf{z} \mid \mathbf{x})=\prod_{k=1}^{n} \prod_{i=1}^{M} p\left(z_{i}^{k} \mid \mathbf{x}^{k}\right) .
$$

Our goal is to locate the target location in real time based on the collection of range measurements $\mathbf{z}$, which is equivalent to calculate the a posteriori distribution of $\mathrm{x}$. Following the Bayes' theorem, we have $p(\mathbf{x} \mid \mathbf{z}) \propto p(\mathbf{x}) p(\mathbf{z} \mid \mathbf{x})$, where $p(\mathbf{x})$ is the joint a priori distribution of the target location, which obeys the Markov chain,

$$
p(\mathbf{x})=p\left(\mathbf{x}^{0}\right) \prod_{k=1}^{n} p\left(\mathbf{x}^{k} \mid \mathbf{x}^{k-1}\right),
$$

where $p\left(\mathbf{x}^{k} \mid \mathbf{x}^{k-1}\right)$ denotes the state transition probability. Assuming that $p\left(\mathrm{x}^{0}\right)$ is the initial a priori information, we have the state transition function as

$$
\mathbf{x}^{k}=\mathbf{x}^{k-1}+\delta_{t} \hat{\mathbf{s}}^{k-1}+\boldsymbol{\Delta}_{s},
$$

where $\hat{\mathbf{s}}^{k-1}=\left[\hat{s}_{x}^{k-1}, \hat{s}_{y}^{k-1}\right]=\left(\hat{\mathbf{x}}^{k-1}-\hat{\mathbf{x}}^{k-2}\right) / \delta_{t}$ denotes the estimated velocity at instant $(k-1), \delta_{t}$ is the time slot duration and $\Delta_{s}$ is transition noise distributed as $\mathcal{N}\left(\boldsymbol{\Delta}_{s}, \mathbf{0}, \mathbf{V}_{s}\right)$. Consequently, we have the factorization of the a posteriori distribution as

$$
p(\mathbf{x} \mid \mathbf{z})=p\left(\mathbf{x}^{0}\right) \prod_{k=1}^{n} p\left(\mathbf{x}^{k} \mid \mathbf{x}^{k-1}\right) \prod_{k=1}^{n} \prod_{i=1}^{M} p\left(z_{i}^{k} \mid \mathbf{x}^{k}\right) .
$$

Generally, we want to obtain the target location at some certain instant $k$ under the MMSE criterion $\hat{\mathbf{x}}^{k}=\mathbb{E}_{\mathbf{x}^{k}}\left(\mathbf{x}^{k} \mid \mathbf{z}\right)$, which requires the marginal distribution of $\mathrm{x}^{k}$. To avoid the exponential complexity of direct marginalization, we resort to the factor graph approach, which is efficient in solving marginalization problems.

\section{Passive Localization With Unified FACTOR GRAPH FRAMEWORK}

\section{A. Factor Graph Representation}

The classic factor graph is comprised of edges representing variables and factor nodes representing functions. An edge is connected to a factor node if and only if the variable is involved in this function. By applying the sum product algorithm (SPA) [35] to the factor graph, the approximate marginal distributions ("belief") of variables are obtained, i.e. $b(x) \simeq p(x \mid z)$. Using the shorthand $f_{i}^{k}$ for the likelihood

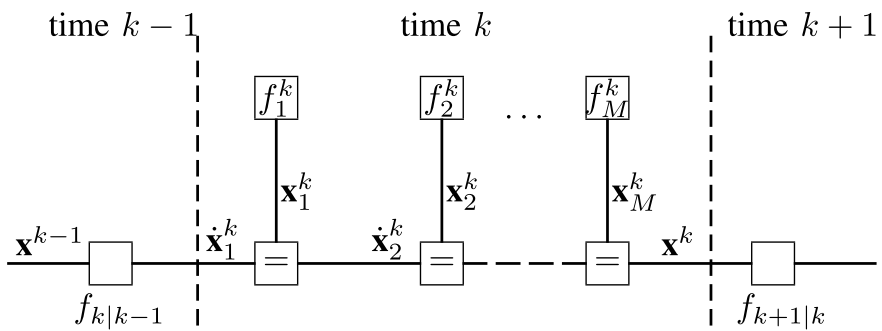

Fig. 2. Factor graph representation for the factorization (7). The node '=' denotes the equality constraint $f_{\delta}$.

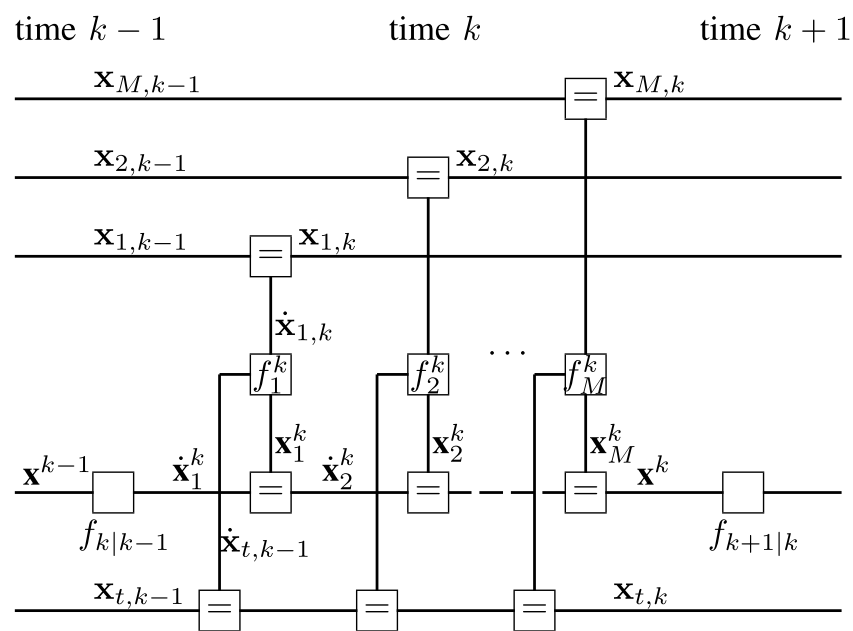

Fig. 3. Factor graph for TOA passive localization with inaccurate transceiver positions.

function (3) and $f_{k \mid k-1}$ for the state transition function, the factor graph representing the factorization in (7) is shown in Fig.2. Since in (Forney-style) factor graphs, there is a unique edge for every variable and that every edge can connect at most two factors. Therefore the equality constraint node $=$ is introduced that allows more than two factors to connect to the same variable.

The belief of target location at instant $k$ is expressed as

$$
b\left(\mathbf{x}^{k}\right)=\mu_{f_{k \mid k-1} \rightarrow \mathbf{x}^{k}}\left(\mathbf{x}^{k}\right) \prod_{i=1}^{M} \mu_{f_{i}^{k} \rightarrow \mathbf{x}_{i}^{k}}\left(\mathbf{x}^{k}\right),
$$

where $\mu_{f \rightarrow x}$ denote the message from $f$ to $x$. Then the estimate of the target location is given by $\hat{\mathbf{x}}^{k}=\mathbb{E}_{\mathbf{x}^{k}}\left[b\left(\mathbf{x}^{k}\right)\right]$.

\section{B. Position Uncertainties of Transceivers}

The positions of transceivers are not always perfectly known. In such situations, they should be inferred from the joint a posteriori distribution $p\left(\mathbf{x}, \mathbf{x}_{t}, \mathbf{x}_{r} \mid \mathbf{z}\right)$. Using a similar factorization to that in (7), the factor graph is modified by adding edges related to the transceivers' positions, which is shown in Fig. 3. Note that the factor graph is no longer loop free, hence an iterative message passing scheme is utilized to compute the belief. For example, upon denoting the iteration index by $l$, the message $\mu_{f_{1}^{k} \rightarrow \mathbf{x}_{1}^{k}}^{(l)}\left(\mathbf{x}_{1}^{k}\right)$ is calculated according 
to the SPA rules as

$$
\mu_{f_{1}^{k} \rightarrow \mathbf{x}_{1}^{k}}^{(l)}\left(\mathbf{x}_{1}^{k}\right)=\iint f_{1}^{k} \mu_{\dot{\mathbf{x}}_{1, k} \rightarrow f_{1}^{k}}^{(l)}\left(\dot{\mathbf{x}}_{1, k}\right) \mu_{\dot{\mathbf{x}}_{t, k-1} \rightarrow f_{1}^{k}}^{(l)}\left(\dot{\mathbf{x}}_{t, k-1}\right)
$$

The integration in (9) can not be carried out analytically due to the nonlinear square root terms in the range measurement. Several approximate methods have been proposed for solving this problem, such as PF [29] and min-divergence approximation [36]. Both methods suffer from high complexity, since a high number of samples are used. In view of the iterative nature of loopy message passing, at instant $k$ and the $l$ th iteration, we perform the first order Taylor expansion on the Euclidean norm based on previous estimated target and transceiver locations,

$$
\begin{aligned}
d_{i}^{k} & =\left\|\mathbf{x}^{k}-\mathbf{x}_{i}\right\|+\left\|\mathbf{x}^{k}-\mathbf{x}_{t}\right\| \\
& \simeq A_{i}^{k,(l-1)} \mathbf{x}^{k}+B_{i}^{k,(l-1)} \mathbf{x}_{i}+C_{i}^{k,(l-1)} \mathbf{x}_{t}+D_{i}^{k,(l-1)},
\end{aligned}
$$

where $\mathbf{A}_{i}^{k,(l-1)}, \mathbf{B}_{i}^{k,(l-1)}, \mathbf{C}_{i}^{k,(l-1)}$ and $D_{i}^{k,(l-1)}$ are given as

$$
\begin{aligned}
\mathbf{A}_{i}^{k,(l-1)}= & \frac{\left(\hat{\mathbf{x}}^{k,(l-1)}-\hat{\mathbf{x}}_{i}^{(l-1)}\right)^{T}}{\left\|\hat{\mathbf{x}}^{k,(l-1)}-\hat{\mathbf{x}}_{i}^{(l-1)}\right\|}+\frac{\left(\hat{\mathbf{x}}^{k,(l-1)}-\hat{\mathbf{x}}_{t}^{(l-1)}\right)^{T}}{\left\|\hat{\mathbf{x}}^{k,(l-1)}-\hat{\mathbf{x}}_{t}^{(l-1)}\right\|} \\
\mathbf{B}_{i}^{k,(l-1)}= & \frac{\left(\hat{\mathbf{x}}_{i}^{(l-1)}-\hat{\mathbf{x}}^{k,(l-1)}\right)^{T}}{\left\|\hat{\mathbf{x}}^{k,(l-1)}-\hat{\mathbf{x}}_{i}^{(l-1)}\right\|} \\
\mathbf{C}_{i}^{k,(l-1)}= & \frac{\left(\hat{\mathbf{x}}_{t}^{(l-1)}-\hat{\mathbf{x}}^{k,(l-1)}\right)^{T}}{\left\|\hat{\mathbf{x}}^{k,(l-1)}-\hat{\mathbf{x}}_{t}^{(l-1)}\right\|} \\
D_{i}^{k,(l-1)}= & \left\|\hat{\mathbf{x}}^{k,(l-1)}-\hat{\mathbf{x}}_{i}^{(l-1)}\right\|+\left\|\hat{\mathbf{x}}^{k,(l-1)}-\hat{\mathbf{x}}_{t}^{(l-1)}\right\| \\
& -\mathbf{A}_{i}^{k,(l-1)} \hat{\mathbf{x}}^{k,(l-1)}-\mathbf{B}_{i}^{k,(l-1)} \hat{\mathbf{x}}_{i}^{(l-1)} \\
& -\mathbf{C}_{i}^{k,(l-1)} \hat{\mathbf{x}}_{t}^{(l-1)} .
\end{aligned}
$$

With the aid of (6) and (10), the passive localization problem can be transformed into a linear state-space model. According to [28], it is straightforward to convert the factor graph into the scheme in Fig. 4. The new factor node $\boxplus$ represents the addition constraint $y=x_{1}+x_{2}$ or $\delta\left(y-x_{1}-x_{2}\right)$, while $A$ denotes the multiplication operation. Based on this factor graph and the Gaussian message passing (GMP) of [28], the marginal distribution of every variable can be efficiently obtained in Gaussian form. The message update rules for three basic node types, namely for the adder node, constant multiplier node and equality node are summarized in [28] and not given in this paper due to space limitation. To simplify the notation, we use $\vec{r}$ to denote the messages that are passed along the arrow direction, while $\leftarrow$ indicates that the message passing direction is the opposite of the arrow direction.

Note that all messages and beliefs in this graph are of Gaussian form. They can be parameterized by the corresponding mean vectors and covariance matrices, e.g. $\vec{\mu}_{\mathbf{x}_{i, k-1}}=$ $\mathcal{N}\left(\mathbf{x}_{i, k-1}, \overrightarrow{\mathbf{m}}_{\mathbf{x}_{i, k-1}}, \overrightarrow{\mathbf{V}}_{\mathbf{x}_{i, k-1}}\right)$. Furthermore, we use weight matrix $\mathbf{W}=\mathbf{V}^{-1}$ for convenience.

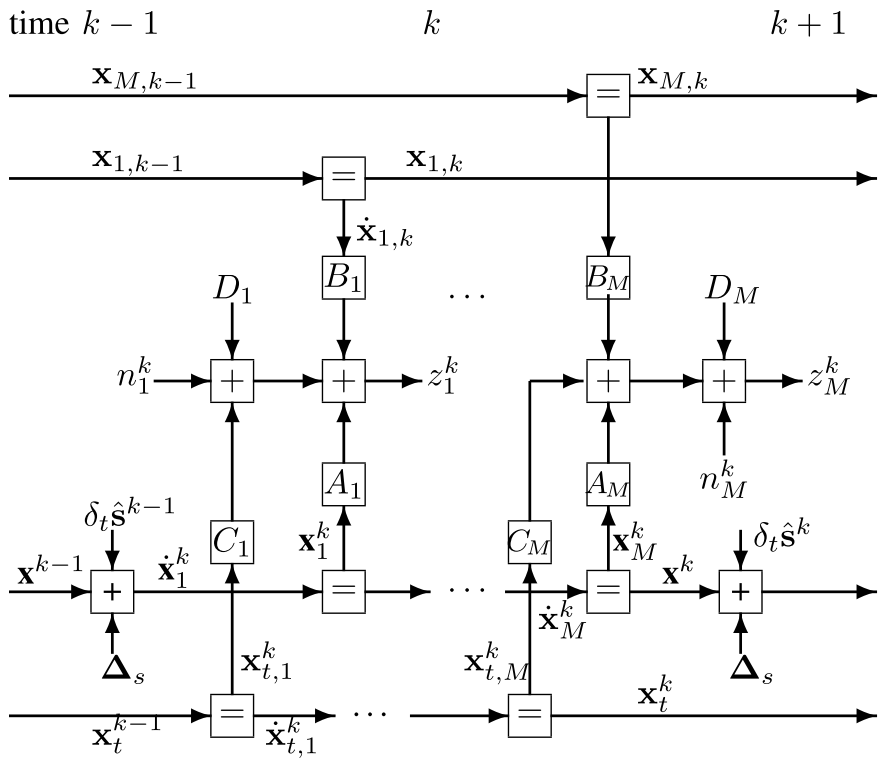

Fig. 4. State space model based factor graph for TOA passive localization with inaccurate transceivers.

- Calculation of beliefs at instant $k$ :

We first focus our attention on calculating $b\left(\mathrm{x}^{k}\right)$, which is the same as the message $\vec{\mu}_{\mathbf{x}^{k}}=\mathcal{N}\left(\mathbf{x}^{k}, \overrightarrow{\mathbf{m}}_{\mathbf{x}^{k}}, \overrightarrow{\mathbf{V}}_{\mathbf{x}^{k}}\right)$ with

$$
\begin{aligned}
\overrightarrow{\mathbf{V}}_{\mathbf{x}^{k}} & =\left(\overrightarrow{\mathbf{W}}_{\dot{\mathbf{x}}_{M}^{k}}+\overleftarrow{\mathbf{W}}_{\mathbf{x}_{M}^{k}}\right)^{-1}=\left(\overrightarrow{\mathbf{W}}_{\dot{\mathbf{x}}_{M-1}^{k}}+\overleftarrow{\mathbf{W}}_{\mathbf{x}_{M-1}^{k}}+\overleftarrow{\mathbf{W}}_{\mathbf{x}_{M}^{k}}\right)^{-1} \\
& =\left(\overrightarrow{\mathbf{W}}_{\dot{\mathbf{x}}_{1}^{k}}+\sum_{i=1}^{M} \overleftarrow{\mathbf{W}}_{\mathbf{x}_{i}^{k}}\right)^{-1} \\
\overrightarrow{\mathbf{m}}_{\mathbf{x}^{k}} & =\overrightarrow{\mathbf{V}}_{\mathbf{x}^{k}}\left(\overrightarrow{\mathbf{W}}_{\dot{\mathbf{x}}^{k}} \overrightarrow{\mathbf{m}}_{\dot{\mathbf{x}}^{k}}+\sum_{i=1}^{M} \overleftarrow{\mathbf{W}}_{\mathbf{x}_{i}^{k}} \overleftarrow{\mathbf{m}}_{\mathbf{x}_{i}^{k}}\right)
\end{aligned}
$$

while $\overleftarrow{\mathbf{m}}_{\mathbf{x}_{i}^{k}}$ and $\overleftarrow{\mathbf{V}}_{\mathbf{x}_{i}^{k}}$ are calculated based on the observations which follow

$$
\begin{aligned}
& \overleftarrow{\mathbf{m}}_{\mathbf{x}_{i}^{k}}=\left(\mathbf{A}_{i}^{k}\right)^{\dagger}\left(z_{i}^{k}-D_{i}^{k}-\mathbf{B}_{i}^{k} \overrightarrow{\mathbf{m}}_{\dot{\mathbf{x}}_{i, k}}-\mathbf{C}_{i}^{k} \overrightarrow{\mathbf{m}}_{\mathbf{x}_{t, i}^{k}}\right) \\
& \overleftarrow{\mathbf{W}}_{\mathbf{x}_{i}^{k}}=\mathbf{A}_{i}^{k, T}\left(\mathbf{B}_{i}^{k} \overrightarrow{\mathbf{V}}_{\dot{\mathbf{x}}_{i, k}} \mathbf{B}_{i}^{k, T}+\mathbf{C}_{i}^{k} \overrightarrow{\mathbf{V}}_{\mathbf{x}_{t, i}^{k}} \mathbf{C}_{i}^{k, T}+\sigma_{i}^{2}\right)^{-1} A_{i}^{k}
\end{aligned}
$$

where $\left(\mathbf{A}_{i}^{k}\right)^{\dagger}=\left(\mathbf{A}_{i}^{k, T} \mathbf{A}_{i}^{k}\right)^{-1} \mathbf{A}_{i}^{k, T}$ is the Moore-Penrose pseudo-inverse of vector $\mathbf{A}_{i}^{k} \cdot \overrightarrow{\mathbf{m}}_{\dot{\mathbf{x}}_{1}^{k}}$ and $\overrightarrow{\mathbf{V}}_{\dot{\mathbf{x}}_{1}^{k}}$ are obtained according to the prediction step. Under the assumption that the message $\vec{\mu}_{\mathbf{x}^{k-1}}$ is available in Gaussian form along with $\overrightarrow{\mathbf{m}}_{x^{k-1}}$ and $\overrightarrow{\mathbf{V}}_{x^{k-1}}, \overrightarrow{\mathbf{m}}_{\dot{\mathbf{x}}^{k}}$ and $\overrightarrow{\mathbf{V}}_{\dot{\mathbf{x}}^{k}}$ are expressed as

$$
\begin{aligned}
& \overrightarrow{\mathbf{m}}_{\dot{\mathbf{x}}_{1}^{k}}=\overrightarrow{\mathbf{m}}_{\mathbf{x}^{k-1}}+\delta_{t} \hat{\mathbf{s}}^{k-1} \\
& \overrightarrow{\mathbf{V}}_{\dot{\mathbf{x}}_{1}^{k}}=\overrightarrow{\mathbf{V}}_{\mathbf{x}^{k-1}}+\mathbf{V}_{s}
\end{aligned}
$$

In (17) and (18), $\overrightarrow{\mathbf{m}}_{\dot{\mathbf{x}}_{i, k}}, \overrightarrow{\mathbf{V}}_{\dot{\mathbf{x}}_{i, k}}, \overrightarrow{\mathbf{m}}_{x_{t, i}^{k}}, \overrightarrow{\mathbf{V}}_{x_{t, i}^{k}}$ are the vector of mean values and the covariance matrices of $\vec{\mu}_{\dot{\mathbf{x}}_{i, k}}, \vec{\mu}_{x_{t, i}^{k}}$, 
yielding

$$
\begin{aligned}
\overrightarrow{\mathbf{m}}_{\dot{\mathbf{x}}_{i, k}} & =\overrightarrow{\mathbf{m}}_{\mathbf{x}_{i, k-1}}, \\
\overrightarrow{\mathbf{V}}_{\dot{\mathbf{x}}_{i, k}} & =\overrightarrow{\mathbf{V}}_{\mathbf{x}_{i, k-1}}, \\
\overrightarrow{\mathbf{m}}_{\mathbf{x}_{t, i}^{k}} & =\overleftarrow{\mathbf{m}}_{\dot{\mathbf{x}}_{t, i}^{k}}+\overrightarrow{\mathbf{m}}_{\dot{\mathbf{x}}_{t, i-1}^{k}}=\overrightarrow{\mathbf{m}}_{\mathbf{x}_{t}^{k-1}}+\sum_{c=1, c \neq i}^{M-1} \overleftarrow{\mathbf{m}}_{\mathbf{x}_{t, c}^{k}} \\
\overrightarrow{\mathbf{V}}_{\mathbf{x}_{t, i}^{k}} & =\overleftarrow{\mathbf{V}}_{\dot{\mathbf{x}}_{t, i}^{k}}+\overrightarrow{\mathbf{V}}_{\dot{\mathbf{x}}_{t, i-1}^{k}}=\overrightarrow{\mathbf{V}}_{\mathbf{x}_{t}^{k-1}}+\sum_{c=1, c \neq i}^{M-1} \overleftarrow{\mathbf{V}}_{\mathbf{x}_{t, c}^{k}}
\end{aligned}
$$

Similarly, when calculating the belief of transmitter location, $\overleftarrow{\mathbf{m}}_{\mathbf{x}_{t, i}^{k}}$ and $\overleftarrow{\mathbf{V}}_{\mathbf{x}_{t, i}^{k}}$ have the form of

$$
\begin{aligned}
\overleftarrow{\mathbf{m}}_{\mathbf{x}_{t, i}^{k}} & =\left(\mathbf{C}_{i}^{k}\right)^{\dagger}\left(z_{i}^{k}-D_{i}^{k}-\mathbf{B}_{i}^{k} \overrightarrow{\mathbf{m}}_{\dot{\mathbf{x}}_{i, k}}-\mathbf{A}_{i}^{k} \overrightarrow{\mathbf{m}}_{\mathbf{x}_{i}^{k}}\right) \\
\overleftarrow{\mathbf{W}}_{\mathbf{x}_{t, i}^{k}} & =\mathbf{C}_{i}^{k, T}\left(\mathbf{B}_{i}^{k} \overrightarrow{\mathbf{V}}_{\dot{\mathbf{x}}_{i, k}} \mathbf{B}_{i}^{k, T}+\mathbf{A}_{i}^{k} \overrightarrow{\mathbf{V}}_{\mathbf{x}_{i}^{k}} \mathbf{A}_{i}^{k, T}+\sigma_{i}^{2}\right)^{-1} \mathbf{C}_{i}^{k}
\end{aligned}
$$

Consequently, the mean and covariance matrix of the message $\vec{\mu}_{\mathbf{x}_{t}^{k}}\left(b\left(\mathbf{x}_{t}^{k}\right)\right)$ can be written as

$$
\begin{aligned}
& \overrightarrow{\mathbf{V}}_{\mathbf{x}_{t}^{k}}=\left(\overrightarrow{\mathbf{W}}_{\mathbf{x}_{t}^{k-1}}+\sum_{i=1}^{M-1} \overleftarrow{\mathbf{W}}_{\mathbf{x}_{t, i}^{k}}\right)^{-1} \\
& \overrightarrow{\mathbf{m}}_{\mathbf{x}_{t}^{k}}=\overrightarrow{\mathbf{V}}_{\mathbf{x}_{t}^{k}}\left(\overrightarrow{\mathbf{W}}_{\mathbf{x}_{t}^{k-1}} \overrightarrow{\mathbf{m}}_{\mathbf{x}_{t}^{k}}+\sum_{i=1}^{M-1} \overleftarrow{\mathbf{W}}_{\mathbf{x}_{t, i}^{k}} \overleftarrow{\mathbf{m}}_{\mathbf{x}_{t, i}^{k}}\right)
\end{aligned}
$$

The belief of receiver $i$ 's position $b\left(\mathbf{x}_{i, k}\right)$ is calculated in the same way as

$$
\begin{aligned}
& \overrightarrow{\mathbf{V}}_{\mathbf{x}_{i, k}}=\left(\overrightarrow{\mathbf{V}}_{\dot{\mathbf{x}}_{i, k-1}}^{-1}+\overleftarrow{\mathbf{W}}_{\dot{\mathbf{x}}_{i, k}}\right)^{-1} \\
& =\left(\overrightarrow{\mathbf{V}}_{\mathbf{x}_{i, k-1}}^{-1}+\mathbf{B}_{i}^{k, T}\left(\mathbf{C}_{i}^{k} \overrightarrow{\mathbf{V}}_{\dot{\mathbf{x}}_{t, i}^{k}} \mathbf{C}_{i}^{k, T}+\mathbf{A}_{i}^{k} \overrightarrow{\mathbf{V}}_{\mathbf{x}_{i}^{k}} \mathbf{A}_{i}^{k, T}+\sigma_{i}^{2}\right)^{-1} \mathbf{B}_{i}^{k}\right)^{-1} \\
& \overrightarrow{\mathbf{m}}_{\mathbf{x}_{i, k}} \\
& =\overrightarrow{\mathbf{V}}_{\mathbf{x}_{i, k}}\left(\overrightarrow{\mathbf{W}}_{\dot{\mathbf{x}}_{i, k}} \overrightarrow{\mathbf{m}}_{\dot{\mathbf{x}}_{i, k}}+\overleftarrow{\mathbf{W}}_{\dot{\mathbf{x}}_{i, k}} \overleftarrow{\mathbf{m}}_{\dot{\mathbf{x}}_{i, k}}\right), \\
& =\overrightarrow{\mathbf{V}}_{\mathbf{x}_{i, k}}\left(\overrightarrow{\mathbf{V}}_{\mathbf{x}_{i, k-1}}^{-1} \overrightarrow{\mathbf{m}}_{\mathbf{x}_{i, k-1}}+\mathbf{B}_{i}^{k, T}\left(\sigma_{i}^{2}+\mathbf{A}_{i}^{k} \overrightarrow{\mathbf{V}}_{\mathbf{x}_{i}^{k}} \mathbf{A}_{i}^{k, T}\right.\right. \\
& \left.\left.\quad+\mathbf{C}_{i}^{k} \overrightarrow{\mathbf{V}}_{\dot{\mathbf{x}}_{t, i}^{k}} \mathbf{C}_{i}^{k, T}\right)^{-1}\left(z_{i}^{k}-D_{i}^{k}-\mathbf{C}_{i}^{k} \overrightarrow{\mathbf{m}}_{\mathbf{x}_{t, i}^{k}}-\mathbf{A}_{i}^{k} \overrightarrow{\mathbf{m}}_{\mathbf{x}_{i}^{k}}\right)\right) .
\end{aligned}
$$

Based on iterative message passing, the beliefs of transceiver and target locations at instant $k$ are determined. Then the location estimate can be obtained by the MMSE estimator.

\section{Asynchronous Receivers}

In this subsection, we consider the TOA passive target localization in a fully asynchronous network. For ease of exposition, the positions of transceivers are assumed to be accurately known. Since there exist clock skew and offset, the signal propagation time (1) observed at receiver $i$ becomes

$$
t_{i}^{k}=\gamma_{i}^{k}\left(\frac{\left\|\mathbf{x}^{k}-\mathbf{x}_{t}\right\|+\left\|\mathbf{x}^{k}-\mathbf{x}_{i}\right\|}{c}+\omega_{i}^{k}\right)+\theta_{i}^{k},
$$

where $\gamma_{i}^{k}$ and $\theta_{i}^{k}$ denote the clock skew and offset of receiver $i$ at instant $k$. For convenience, we use the definition $\alpha_{i}=1 / \gamma_{i}$

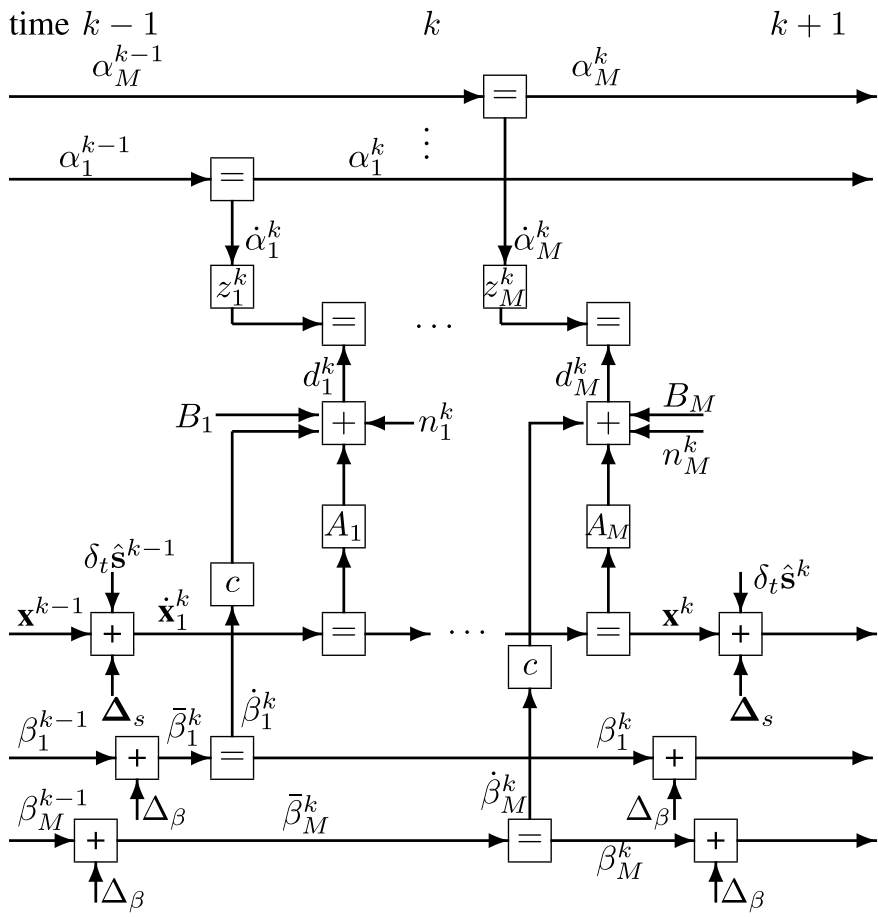

Fig. 5. Factor graph for TOA passive localization in time-varying asynchronous networks.

and $\beta_{i}^{k}=\theta_{i}^{k} / \gamma_{i}$. In this work, it is assumed that the clock skew is static $\alpha_{i}^{k}=\alpha_{i}^{k-1}=1 / \gamma_{i}$, while $\beta_{i}^{k}$ varies according to a Markov evolution model [37], [38] ${ }^{6}$

$$
\beta_{i}^{k}=\beta_{i}^{k-1}+\Delta_{\beta}
$$

where we have $\Delta_{\beta} \sim \mathcal{N}\left(0, V_{\beta}\right)$. The related range measurement is given by,

$$
\alpha_{i} z_{i}^{k}=\left\|\mathbf{x}^{k}-\mathbf{x}_{t}\right\|+\left\|\mathbf{x}^{k}-\mathbf{x}_{i}\right\|+c \beta_{i}^{k}+n_{i}^{k} .
$$

We also utilize the Taylor expansion to linearize the nonlinear terms around the previous estimate of target location. Accordingly, (33) is rewritten as

$$
\alpha_{i} z_{i}^{k}=\mathbf{A}_{i}^{k,(l-1)} \mathbf{x}+B_{i}^{k,(l-1)}+c \beta_{i}^{k}+n_{i}^{k}
$$

with

$$
\begin{aligned}
\mathbf{A}_{i}^{k,(l-1)}= & \frac{\left(\hat{\mathbf{x}}^{k,(l-1)}-\mathbf{x}_{i}\right)^{T}}{\left\|\hat{\mathbf{x}}^{k,(l-1)}-\mathbf{x}_{i}\right\|}+\frac{\left(\hat{\mathbf{x}}^{k,(l-1)}-\mathbf{x}_{t}\right)^{T}}{\left\|\hat{\mathbf{x}}^{k,(l-1)}-\mathbf{x}_{t}\right\|} \\
B^{k,(l-1)}= & \left\|\hat{\mathbf{x}}^{k,(l-1)}-\mathbf{x}_{i}\right\|+\left\|\hat{\mathbf{x}}^{k,(l-1)}-\mathbf{x}_{t}\right\| \\
& -\mathbf{A}_{i}^{k,(l-1)} \hat{\mathbf{x}}^{k,(l-1)}
\end{aligned}
$$

Based on the linear state-space model (6), (32) and (34), the factor graph for our asynchronous network is depicted in Fig. 5. Since we have formulated the calculations of messages explicitly in the previous subsection, here only the

${ }^{6}$ In reality, the clock skew is time-variant. However the variation is generally small and can be ignored. 
beliefs of variables are derived, i.e.

$$
\begin{aligned}
& \overrightarrow{\mathbf{V}}_{\mathbf{x}^{k}} \\
& =\left(\overrightarrow{\mathbf{W}}_{\dot{\mathbf{x}}_{1}^{k}}+\sum_{i=1}^{M} \frac{\mathbf{A}_{i}^{k, T} \mathbf{A}_{i}^{k}}{c^{2} \vec{V}_{\dot{\beta}_{i, k}}+\left(z_{i}^{k}\right)^{2} \vec{V}_{\dot{\alpha}_{i}^{k}}+\sigma_{i}^{2}}\right)^{-1}, \\
& \overrightarrow{\mathbf{m}}_{\mathbf{x}^{k}}=\overrightarrow{\mathbf{V}}_{\mathbf{x}^{k}} \\
& \cdot\left(\overrightarrow{\mathbf{W}}_{\dot{\mathbf{x}}^{k}} \overrightarrow{\mathbf{m}}_{\dot{\mathbf{x}}^{k}}+\sum_{i=1}^{M} \frac{\mathbf{A}_{i}^{k, T}\left(\vec{m}_{\dot{\alpha}_{i}^{k}} z_{i}^{k}-B_{i}^{k}-c \vec{m}_{\dot{\beta}_{i, k}}\right)}{c^{2} \vec{V}_{\dot{\beta}_{i, k}}+\left(z_{i}^{k}\right)^{2} \vec{V}_{\dot{\alpha}_{i}^{k}}+\sigma_{i}^{2}}\right), \\
& \vec{V}_{\alpha_{i}^{k}} \\
& =\left(\frac{1}{\vec{V}_{\alpha^{k-1}}}+\frac{\left(z_{i}^{k}\right)^{2}}{\mathbf{A}_{i}^{k} \overrightarrow{\mathbf{V}}_{\mathbf{x}_{i}^{k}} \mathbf{A}_{i}^{k, T}+c^{2} \vec{V}_{\dot{\beta}_{i, k}}+\sigma_{i}^{2}}\right)^{-1}, \\
& \vec{m}_{\alpha_{i}^{k}} \\
& =\vec{V}_{\alpha^{k}}\left(\frac{\vec{m}_{\alpha^{k-1}}}{\vec{V}_{\alpha^{k-1}}}+\frac{z_{i}^{k} \cdot\left(\mathbf{A}_{i}^{k} \overrightarrow{\mathbf{m}}_{\mathbf{x}_{i}^{k}}+c \vec{m}_{\dot{\beta}_{i, k}}+B_{i}^{k}\right)}{\mathbf{A}_{i}^{k} \overrightarrow{\mathbf{V}}_{\mathbf{x}_{i}^{k}} \mathbf{A}_{i}^{k, T}+c^{2} \vec{V}_{\dot{\beta}_{i, k}}+\sigma_{i}^{2}}\right), \\
& \vec{V}_{\beta_{i}^{k}} \\
& =\left(\frac{1}{\vec{V}_{\beta_{i}^{k-1}}+V_{\beta}}+\frac{c^{2}}{\mathbf{A}_{i}^{k} \overrightarrow{\mathbf{V}}_{\mathbf{x}_{i}^{k}} \mathbf{A}_{i}^{k, T}+\left(z_{i}^{k}\right)^{2} \vec{V}_{\dot{\alpha}_{i}^{k}}+\sigma_{i}^{2}}\right)^{-1}, \\
& \vec{m}_{\beta_{i}^{k}}=\overrightarrow{\mathbf{V}}_{\beta_{i}^{k}} \\
& \cdot\left(\frac{\vec{m}_{\beta_{i}^{k-1}}}{\vec{V}_{\beta_{i}^{k-1}}+V_{\beta}}+\frac{c \cdot\left(\vec{m}_{\dot{\alpha}_{i}^{k}} z_{i}^{k}-B_{i}^{k}-\mathbf{A}_{i}^{k} \overrightarrow{\mathbf{m}}_{\mathbf{x}_{i}^{k}}\right)}{\mathbf{A}_{i}^{k} \overrightarrow{\mathbf{V}}_{\mathbf{x}_{i}^{k}} \mathbf{A}_{i}^{k, T}+\left(z_{i}^{k}\right)^{2} \vec{V}_{\dot{\alpha}_{i}^{k}}+\sigma_{i}^{2}}\right) .
\end{aligned}
$$

The estimates of variables can be determined with the aid of beliefs. Note that $\alpha_{i}, \beta_{i}^{k}$ are transformed from the true clock skew and offset, thus the estimates of clock skew and offset become:

$$
\begin{aligned}
& \hat{\gamma}_{i}=\frac{1}{\hat{\alpha}_{i}}, \\
& \hat{\theta}_{i}^{k}=\frac{\hat{\beta}_{i}^{k}}{\hat{\alpha}_{i}} .
\end{aligned}
$$

\section{Outliers}

The main sources of outliers occurring in WSNs include sensor errors and malicious attacks. The malicious attacks form part of network security while sensor errors are typically caused by faulty sensors. Finally, outliers may also be caused by deleterious phenomena that affect the observations, but generally, outliers caused by errors occur more frequently than those by events. Thus in this paper we only consider the outliers caused by sensor detection failure in passive localization.

At instant $k$, the $i$ th receiver makes an observation $z_{i}^{k}$. If the sensor fails to detect the TOA signal, only the measurement noise is observed. By contrast, if the receiver is working as

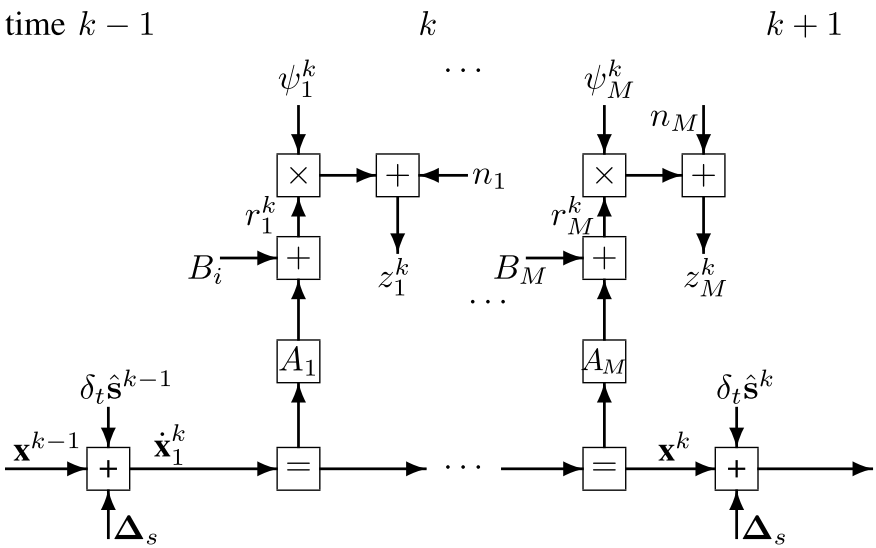

Fig. 6. Factor graph for joint TOA passive localization and outliers detection.

intended, the range measurement is observed, following

$$
z_{i}^{k}= \begin{cases}\left\|\mathbf{x}^{k}-\mathbf{x}_{t}\right\|+\left\|\mathbf{x}^{k}-\mathbf{x}_{i}\right\|+n_{i}^{k} & \text { Functioning } \\ n_{i}^{k} & \text { Outliers }\end{cases}
$$

where $\tilde{n}_{i}^{k}$ obeys a half-normal distribution formulated as $\sqrt{\frac{2}{\sigma_{i}^{2} \pi}} \exp \left(-\left(\tilde{n}_{i}^{k}\right)^{2} / 2 \sigma_{i}^{2}\right), \tilde{n}_{i}^{k}>0$. For brevity, in the algorithm design, we assume $n_{i}^{k}=\tilde{n}_{i}^{k}>0$.

Denote the occurrence probability of outliers by $p_{o}$, then the likelihood function is rewritten as

$$
\begin{aligned}
p\left(z_{i}^{k} \mid \mathbf{x}\right) \propto\left(1-p_{o}\right) \cdot \exp \left(-\frac{\left(z_{i}^{k}-d_{i}^{k}\right)^{2}}{2 \sigma_{i}^{2}}\right) & \\
& +p_{o} \cdot \exp \left(-\frac{\left(z_{i}^{k}\right)^{2}}{\sigma_{i}^{2}}\right) .
\end{aligned}
$$

By introducing a binary state $\psi_{i}^{k}=\{0,1\}$, the observation $z_{i}^{k}$ can be rewritten as

$$
z_{i}^{k}=\psi_{i}^{k}\left(\left\|\mathbf{x}^{k}-\mathbf{x}_{t}\right\|+\left\|\mathbf{x}^{k}-\mathbf{x}_{i}\right\|\right)+n_{i}^{k},
$$

where $\psi_{i}^{k}=1$ indicates that the $i$ th receiver is functioning, while $\psi_{i}^{k}=0$ indicates malfunctioning. The a priori distribution of $\psi_{i}^{k}$ obeys the Bernoulli distribution of

$$
p\left(\psi_{i}^{k}\right)=p_{o}^{1-\psi_{i}^{k}}\left(1-p_{o}\right)^{\psi_{i}^{k}} .
$$

As before, we linearize the range measurement based on Taylor expansion as

$$
z_{i}^{k}=\psi_{i}^{k}\left(\mathbf{A}_{i}^{k,(l-1)} \mathbf{x}+B_{i}^{k,(l-1)}\right)+n_{i}^{k},
$$

with $\mathbf{A}_{i}^{k,(l-1)}$ and $B_{i}^{k,(l-1)}$ previously defined in (35) and (36). Based on (6) and (49), we have the linear state space model and construct the corresponding factor graph, as depicted in Fig. 6. Here we use a multiplier node $\square$ to denote the multiplication of two unknown variables. In order to apply GMP, we employ expectation propagation (EP) [39] to approximate the discrete variable $\psi_{i}^{k}$ by a Gaussian variable. With the assumption that $\overleftarrow{m}_{\psi_{i}^{k}}$ and $\overleftarrow{V}_{\psi_{i}^{k}}$ are available from the previous iteration, we have the mean and variance of the 
belief $b\left(\psi_{i}^{k}\right)$ as

$$
\begin{aligned}
m_{\psi_{i}^{k}} & =\left(1-p_{o}\right) \frac{1}{\sqrt{2 \pi \overleftarrow{V}_{\psi_{i}^{k}}}} \exp \left(-\frac{\left(1-\overleftarrow{m}_{\psi_{i}^{k}}\right)^{2}}{2 \overleftarrow{V}_{\psi_{i}^{k}}}\right) \\
V_{\psi_{i}^{k}} & =m_{\psi_{i}^{k}}-m_{\psi_{i}^{k}}^{2} .
\end{aligned}
$$

Then $\vec{m}_{\psi_{i}^{k}}$ and $\vec{V}_{\psi_{i}^{k}}$ are given as

$$
\begin{aligned}
\vec{V}_{\psi_{i}^{k}} & =\frac{V_{\psi_{i}^{k}} \overleftarrow{V}_{\psi_{i}^{k}}}{V_{\psi_{i}^{k}}-\overleftarrow{V}_{\psi_{i}^{k}}} \\
\vec{m}_{\psi_{i}^{k}} & =\frac{m_{\psi_{i}^{k}} V_{\psi_{i}^{k}}-\overleftarrow{m}_{\psi_{i}^{k}} \overleftarrow{V}_{\psi_{i}^{k}}}{V_{\psi_{i}^{k}}-\overleftarrow{V}_{\psi_{i}^{k}}}
\end{aligned}
$$

However, for the multiplier node $z=x y$, even if the messages $\vec{\mu}_{x}$ and $\overleftarrow{\mu}_{z}$ are Gaussian, we are not able to determine message $\vec{\mu}_{y}$ of a Gaussian form. To this end, we employ the variational message passing (VMP) [40] instead of the SPA at the multiplier node, as derived in Appendix. A. Using VMP, the Gaussian messages for the multiplier node are given by

$$
\begin{aligned}
\overleftarrow{V}_{\psi_{i}^{k}} & =\frac{\sigma_{i}^{2}}{V_{r_{i}^{k}}+m_{r_{i}^{k}}^{2}} \\
\overleftarrow{m}_{\psi_{i}^{k}} & =\frac{z_{i}^{k} m_{r_{i}^{k}}}{V_{r_{i}^{k}}+m_{r_{i}^{k}}^{2}}
\end{aligned}
$$

with

$$
\begin{aligned}
V_{r_{i}^{k}} & =\overleftarrow{V}_{r_{i}^{k}}+\vec{V}_{r_{i}^{k}}, \\
m_{r_{i}^{k}} & =V_{r_{i}^{k}}^{-1}\left(\overleftarrow{W}_{r_{i}^{k}} \overleftarrow{m}_{r_{i}^{k}}+\vec{W}_{r_{i}^{k}} \vec{m}_{r_{i}^{k}}\right) .
\end{aligned}
$$

$V_{r_{i}^{k}}$ and $m_{r_{i}^{k}}$ are updated in a similar way. Then the belief of the target location is obtained as

$$
\begin{aligned}
\overrightarrow{\mathbf{V}}_{\mathbf{x}^{k}} & =\left(\overrightarrow{\mathbf{W}}_{\dot{\mathbf{x}}_{1}^{k}}+\sum_{i=1}^{M} \overleftarrow{\mathbf{W}}_{\mathbf{x}_{i}^{k}}\right)^{-1} \\
& =\left(\overrightarrow{\mathbf{W}}_{\dot{\mathbf{x}}_{1}^{k}}+\sum_{i=1}^{M} \frac{\mathbf{A}_{i}^{k, T} \mathbf{A}_{i}^{k}}{\overleftarrow{V}_{r_{i}^{k}}}\right)^{-1}, \\
\overrightarrow{\mathbf{m}}_{\mathbf{x}^{k}} & =\overrightarrow{\mathbf{V}}_{\mathbf{x}^{k}}\left(\overrightarrow{\mathbf{W}}_{\dot{\mathbf{x}}^{k}} \overrightarrow{\mathbf{m}}_{\dot{\mathbf{x}}^{k}}+\sum_{i=1}^{M} \frac{\mathbf{A}_{i}^{k, T} \overleftarrow{m}_{r_{i}^{k}}}{\overleftarrow{V}_{r_{i}^{k}}}\right)
\end{aligned}
$$

Finally, having $\overleftarrow{m}_{\psi_{i}^{k}}$ and $\overleftarrow{V}_{\psi_{i}^{k}}$, we can estimate the state of $\psi_{i}^{k}$ based on the log-likelihood ratio (LLR) of

$$
\operatorname{LLR}\left(\psi_{i}^{k}\right)=\frac{1-2 \overleftarrow{m}_{\psi_{i}^{k}}}{\overleftarrow{V}_{\psi_{i}^{k}}}+\ln \frac{1-p_{o}}{p_{o}}
$$

For $\operatorname{LLR}\left(\psi_{i}^{k}\right)<0$, we say that the measurement $z_{i}^{k}$ represents an outlier and vice versa.

It can be seen that for all three scenarios, the messages on factor graph are characterized by their means and variances. Therefore the complexity of the proposed algorithm only increases linearly with the number of receivers, i.e., it is on the order of $\mathcal{O}(M)$. By contrast, the PF-based method utilizes $R$ particles and their associated weights to represent the messages, hence resulting in a complexity of $\mathcal{O}(M R)$, which shows the superiority of the proposed algorithm. Moreover, an extension of the factor graph framework to a more complex passive localization problem which combines above three problems or even more becomes straightforward. Gaussian messages can also be derived using simple manipulations at a low complexity. However, this is beyond the scope of this paper.

\section{Distributed Processing}

Distributed detection and estimation have attracted substantial research attention since only local processing and local communications with the immediate neighboring nodes are needed. This is attractive for resource-constrained WSNs. In this section, we propose a low complexity yet efficient distributed algorithm for passive localization.

The most prominent method in distributed localization is the likelihood consensus of [41], which aims for obtaining the global likelihood function at each receiver in a distributed fashion as $\operatorname{LC}\left\{p\left(z_{1}^{k} \mid \mathbf{x}^{k}\right), \ldots, p\left(z_{M}^{k} \mid \mathbf{x}^{k}\right)\right\}=p\left(\mathbf{z}^{k} \mid \mathbf{x}^{k}\right)$. After evaluating the likelihood consensus for a few iterations, all receivers acquire knowledge from the other receivers in the network. Then the target location can be estimated individually by all the receivers.

Due to the nonlinear square root term in (2), the likelihood consensus is usually evaluated using distributed particle filtering (DPF). The main drawback of DPF is its high complexity and high communication. overhead imposed by the exchange of a large number of packets between the neighboring receivers Moreover, since we introduce variables related to the clock offset and to other transceiver-induced uncertainties, the particles should be sampled based on an extended space, which further increases both the communication overhead and the complexity.

Observe from (15) and (16) that the mean and weight matrix of the target location are constituted by the summation of several local parameters obtained by each local receiver. Therefore we aims for reaching consensus concerning the mean and covariance matrix of the variable's belief instead of the global likelihood function. Since only the means and covariance matrices have to be exchanged amongst the neighboring receivers, ${ }^{7}$ we have a so-called parametric consensus process. Accordingly, the communication overhead and computational complexity can be significantly reduced.

Let us assume that both the belief of target location at instant $(k-1)$ and the state transition function are known for all receivers. Then, based on the local observation $z_{i}^{k}$ at instant $k$, the local parameters $\overleftarrow{\mathbf{m}}_{i, \mathbf{x}^{k}}$ (local mean) and weight matrix $\overleftarrow{\mathbf{W}}_{i, \mathbf{x}^{k}}$ (local weight) with respect to the target location are determined at the $i$ th receiver. By applying the consensus process of [25] among the receivers, $\overrightarrow{\mathbf{m}}_{\mathbf{x}^{k}}$ and $\overrightarrow{\mathbf{V}}_{\mathbf{x}^{k}}$ are obtained at each receiver. The consensus process is

\footnotetext{
${ }^{7}$ The word "neighboring" means two receivers can exchange information with each other. It was shown in [25] that the consensus based distributed algorithm is guaranteed to converge, provided that all receivers have at least one neighbor.
} 
TABLE I

Consensus Process For Distributed PASSIVE Localization

\section{Notation:}

$p$ : the consensus iteration index,

$\mathcal{S}_{i}$ : the set of receivers the $i$ th receiver can communicate with, $S_{i}=\left|\mathcal{S}_{i}\right|$ : the number of receiver $i$ 's neighbors,

$\xi=\max _{i} 1 / S_{i}$ : the consensus update rate,

$\mathbf{W}_{i}^{(p)}, \mathbf{W}_{i} \mathbf{m}_{i}^{(p)}$ : the local weight matrix and local transformed mean vector at receiver $i$ in the $p$ th consensus iteration.

\section{At instant $k$}

\section{For all $i$}

Initialization at $p=0$ :

$$
\begin{gathered}
\mathbf{W}_{i}^{(0)}=\overleftarrow{\mathbf{W}}_{\mathbf{x}_{i}^{k}} \\
\mathbf{W}_{i} \mathbf{m}_{i}^{(0)}=\overleftarrow{\mathbf{W}}_{i, \mathbf{x}^{k}} \overleftarrow{\mathbf{m}}_{i, \mathbf{x}^{k}}
\end{gathered}
$$

for $p=1: N_{c}$

\section{end}

$$
\begin{gathered}
\mathbf{W}_{i}^{(p)}=\left(1-\xi S_{i}\right) \mathbf{W}_{i}^{(p-1)}+\xi \sum_{j \in \mathcal{S}_{i}} \mathbf{W}_{i}^{(p-1)} \\
\mathbf{W}_{i} \mathbf{m}_{i}^{(p)}=\left(1-\xi S_{i}\right) \mathbf{W}_{i} \mathbf{m}_{i}^{(p-1)}+\xi \sum_{j \in \mathcal{S}_{i}} \mathbf{W}_{j} \mathbf{m}_{j}^{(p-1)}(64)
\end{gathered}
$$

After $N_{c}$ consensus iterations, the summations of local weight matrices and transformed means are approximated by

$$
\begin{gathered}
\sum_{i=1}^{M} \overleftarrow{\mathbf{W}}_{\mathbf{x}_{i}^{k}}=M \cdot \mathbf{W}_{i}^{\left(N_{c}\right)} \\
\sum_{i=1}^{M} \overleftarrow{\mathbf{W}}_{\mathbf{x}_{i}^{k}} \overleftarrow{\mathbf{m}}_{\mathbf{x}_{i}^{k}}=M \cdot \mathbf{W}_{i} \mathbf{m}_{i}^{\left(N_{c}\right)}
\end{gathered}
$$

Substituting (65) and (66) into (15) and (16), the belief of target location at instant $k$ is determined at each local receiver.

summarized in Table I. ${ }^{8}$ Other unknown variables can also be obtained distributively in the same way.

\section{CRAMÉr-RAO LOWER Bound}

The Cramér-Rao bound (CRB) establishes the lower bound of the variance of an estimator [43]. By following the CRB theorem, the parameters $\tau$ to be estimated obey

$$
\operatorname{cov}(\hat{\boldsymbol{\tau}}) \succeq \mathbf{F}^{-1}
$$

where $\mathbf{F}$ denotes the Fisher information matrix (FIM), ${ }^{9}$ formulating as [43]

$$
\begin{aligned}
\mathbf{F} & =-\mathbb{E}\left[\nabla_{\boldsymbol{\tau}}\left\{\nabla_{\boldsymbol{\tau}}\left(\ln p\left(\boldsymbol{\tau} \mid \mathbf{z}^{k}\right)\right)\right\}\right] \\
& =\underbrace{-\mathbb{E}\left[\nabla_{\boldsymbol{\tau}}\left\{\nabla_{\boldsymbol{\tau}}\left(\ln p\left(\mathbf{z}^{k} \mid \boldsymbol{\tau}\right)\right)\right\}\right]}_{\mathbf{F}_{\mathrm{o}}}-\underbrace{\mathbb{E}\left[\nabla_{\boldsymbol{\tau}}\left\{\nabla_{\boldsymbol{\tau}}(\ln p(\boldsymbol{\tau}))\right\}\right]}_{\mathbf{F}_{\mathrm{p}}},
\end{aligned}
$$

\footnotetext{
${ }^{8}$ Here we use the standard consensus method in which the update rate $\xi$ stays same for all links. There are some other consensus methods utilizing Metropolis weight and gossip algorithm, e.g., [41], [42] and references therein.

${ }^{9}$ In this section, the CRB at time instant $k$ is analyzed for example, since the localization system is time-variant. For simplicity, The superscript $k$ is omitted.
}

with $\mathbf{F}_{\mathrm{o}}$ and $\mathbf{F}_{\mathrm{p}}$ being the Fisher "information" gleaned from observations at instant $k$ and from the previous state. Considering the vector $\tau=\left[\tau_{1}, \ldots, \tau_{N}\right]^{T}$ of $N$ parameters to be estimated, $\mathbf{F}_{\mathrm{p}}$ is an $N \times N$ diagonal matrix

$$
\mathbf{F}_{\mathrm{p}}=\operatorname{diag}\left\{1 / \sigma_{\tau_{1}}^{2}, \ldots, 1 / \sigma_{\tau_{N}}^{2}\right\}
$$

in which the variance $\sigma_{\tau_{n}}$ is related to the variance of the previous time slot and the state transition noise. $\mathbf{F}_{1}$ is also an $N \times N$ matrix, which will be computed in detail in the following.

\section{A. Inaccurate Transceivers}

In a passive localization system relying on $M$ inaccurate receivers, an inaccurate transmitter and a target, the vector of unknown variables is denoted as $\tau=\left[x, y, x_{t}, y_{t}, x_{1}\right.$, $\left.y_{1}, \ldots, x_{M}, y_{M}\right]^{T}$. Then $\mathbf{F}_{\mathrm{o}}$ can be expressed as

$$
\mathbf{F}_{\mathrm{o}}=\mathbf{J} \boldsymbol{\Phi}^{-1} \mathbf{J}^{T}
$$

where $\boldsymbol{\Phi}$ is diagonal with the $i$ th entry being $\sigma_{i}^{2}$, and $\mathbf{J}$ is a $(4+2 M) \times M$ Jacobian matrix with the element of the $i$ th row and the $j$ th column satisfying

$$
J_{i j}=\frac{\partial\left(\left\|\mathbf{x}-\mathbf{x}_{t}\right\|+\left\|\mathbf{x}-\mathbf{x}_{j}\right\|\right)}{\partial \tau_{i}},
$$

For example:

$$
\begin{aligned}
& J_{11}=\frac{x-x_{t}}{\sqrt{\left(x-x_{t}\right)^{2}+\left(y-y_{t}\right)^{2}}}+\frac{x-x_{1}}{\sqrt{\left(x-x_{1}\right)^{2}+\left(y-y_{1}\right)^{2}}}, \\
& J_{21}=\frac{y-y_{t}}{\sqrt{\left(x-x_{t}\right)^{2}+\left(y-y_{t}\right)^{2}}}+\frac{y-y_{1}}{\sqrt{\left(x-x_{1}\right)^{2}+\left(y-y_{1}\right)^{2}}} .
\end{aligned}
$$

Based on $\mathbf{F}_{\mathrm{o}}$ and $\mathbf{F}_{\mathrm{p}}$, the FIM $\mathbf{F}$ can be expressed after some straightforward manipulations as

$$
\mathbf{F}=\left[\begin{array}{ccc}
\mathbf{F}_{x x} & \mathbf{F}_{x x_{t}} & \mathbf{F}_{x x_{i}} \\
\mathbf{F}_{x_{t} x} & \mathbf{F}_{x_{t} x_{t}} & \mathbf{F}_{x_{t} x_{i}} \\
\mathbf{F}_{x_{i} x} & \mathbf{F}_{x_{i} x_{t}} & \mathbf{F}_{x_{i} x_{i}}
\end{array}\right]
$$

where the main diagonal block element is the "information" for the target, transmitter and receiver, respectively. The offdiagonal block elements are referred to as the "cross information" [44]. According to (67), obtaining the CRB requires the inversion of matrix $\mathbf{F}$, which leads to a prohibitive complexity. Moreover, the condition number of the matrix $\mathbf{F}$ will usually be higher when $\mathbf{F}$ is high-dimensional, which may lead to inaccurate numerical computing result. Hence, we employ the equivalent FIM (EFIM) for reducing the dimension of $\mathbf{F}$, while retaining all information of interest [45]. For example, if we mainly focus on the $\mathrm{CRB}$ of target location, based on notations $\mathbf{F}_{t r}=\left[\begin{array}{ll}\mathbf{F}_{x x_{t}} & \mathbf{F}_{x x_{i}}\end{array}\right]$ and $\mathbf{F}_{r}=\left[\begin{array}{ll}\mathbf{F}_{x_{t} x_{t}} & \mathbf{F}_{x_{t} x_{i}} \\ \mathbf{F}_{x_{i} x_{t}} & \mathbf{F}_{x_{i} x_{i}}\end{array}\right]$, the EFIM of target location $\mathbf{F}(\mathbf{x})$ is given by,

$$
\mathbf{F}(\mathbf{x})=\mathbf{F}_{x x}-\mathbf{F}_{t r} \mathbf{F}_{r}^{-1} \mathbf{F}_{t r}^{T}
$$

Then the CRB of target location is calculated as $\operatorname{cov}(\hat{\mathbf{x}}) \succeq \mathbf{F}(\mathbf{x})^{-1}$. Similarly, the CRB of the transceiver locations can also be obtained by the corresponding EFIM. 


\section{B. Clock Skew and Offset}

In asynchronous passive localization networks, the parameters turn out to be the target location and clock parameters $\boldsymbol{\tau}=\left[x, y, \alpha_{1}, \ldots, \alpha_{M}, \beta_{1}, \ldots, \beta_{M}\right]^{T} . \mathbf{F}_{1}$ also satisfies $\mathbf{F}_{1}=\mathbf{J} \boldsymbol{\Phi}^{-1} \mathbf{J}^{T}$, with

$$
\mathbf{J}=\left[\begin{array}{ccc}
J_{11} & \cdots & J_{1 M} \\
J_{21} & \cdots & J_{2 M} \\
& \operatorname{diag}\left\{z_{1}^{k}, \ldots, z_{M}^{k}\right\} & \\
& \operatorname{diag}\left\{c \cdot \mathbf{1}_{M}\right\} &
\end{array}\right]_{(2 M+2) \times M}
$$

Similar to the previous subsection, the FIM $\mathbf{F}$ of passive localization in asynchronous networks is expressed as a block matrix. The EFIM can be invoked for reducing the dimension and for determining the CRB of both the target location and the clock parameters. Note that the clock parameters do not represent the true clock skew and offset. By invoking the Jacobian transformation of [46], if the CRB of $\left[\alpha_{i}, \beta_{i}\right]^{T}$ is given by $\operatorname{cov}\left[\alpha_{i}, \beta_{i}\right]^{T} \succeq \mathbf{F}^{-1}\left(\alpha_{i}, \beta_{i}\right)$, the CRB of the clock parameters of receiver $i$ can be expressed as

$$
\operatorname{cov}\left[\begin{array}{c}
\gamma_{i} \\
\theta_{i}
\end{array}\right] \succeq \Psi^{T} \mathbf{F}^{-1}\left(\alpha_{i}, \beta_{i}\right) \Psi
$$

with $\Psi=\left(\frac{\partial\left[1 / \alpha_{i}, \beta_{i} / \alpha_{i}\right]}{\partial\left[\alpha_{i}, \beta_{i}\right]^{T}}\right)=\left[\begin{array}{cc}-\frac{1}{\alpha_{i}^{2}} & -\frac{\beta_{i}}{\alpha_{i}^{2}} \\ 0 & \frac{1}{\alpha_{i}}\end{array}\right]$.

\section{Outliers}

We aim now for deriving the CRB of target location $\mathbf{x}=[x, y]^{T}$ in the presence of faulty sensors. Since the probability $p_{o}$ of outlier occurrence varies, the CRB is different for different values of $p_{o}$. Since the measurement noise is indepedent, we have

$$
\mathbf{F}_{1}=\mathbb{E}\left[\frac{\partial^{2} \ln \prod_{i} p\left(z_{i} \mid \mathbf{x}\right)}{\partial \mathbf{x}^{2}}\right]=\sum_{i=1}^{M} \mathbb{E}\left[\frac{\partial^{2} \ln p\left(z_{i} \mid \mathbf{x}\right)}{\partial \mathbf{x}^{2}}\right]
$$

Upon considering the calculation of $\frac{\partial^{2} \ln p\left(z_{i} \mid \mathbf{x}\right)}{\partial \mathbf{x}^{2}}$, with (46) and the shorthand notation of $f\left(z_{i}\right)=-\frac{\left(z_{i}-d_{i}\right)^{2}}{\sigma_{i}^{2}}, f\left(n_{i}\right)=-\frac{z_{i}^{2}}{\sigma_{i}^{2}}$ and const $=-\ln \sqrt{2 \pi \sigma_{i}^{2}}$, we have

$$
\begin{aligned}
\ln p\left(z_{i} \mid \mathbf{x}\right) & =\text { const }+\ln \left(\left(1-p_{o}\right) e^{f\left(z_{i}\right)}+p_{o} e^{f\left(n_{i}\right)}\right), \\
& =\text { const }+\ln \left(e^{f\left(z_{i}\right)+\ln \left(1-p_{o}\right)}+e^{f\left(n_{i}\right)+\ln p_{o}}\right) .
\end{aligned}
$$

Note that $\mathbf{F}_{1}$ of (76) cannot be expressed analytically, thus numerical methods have to be used to calculate $\mathbf{F}_{1}$. In order to derive an analytical FIM, we invoke the Jacobian logarithm to simplify (77). It is widely exploited that for real numbers $a_{1}$ and $a_{2}$

$$
\ln \left(e^{a_{1}}+e^{a_{2}}\right)=\max \left\{a_{1}, a_{2}\right\}+\ln \left(1+e^{-\left|a_{1}-a_{2}\right|}\right) .
$$

Generally, for large $\left|a_{1}-a_{2}\right|$, we have $\ln \left(1+e^{-\left|a_{1}-a_{2}\right|}\right) \approx 0$. Consequently, (77) is approximated by

$$
\begin{aligned}
& \ln p\left(z_{i} \mid \mathbf{x}\right) \\
& \quad \approx \text { const }+\max \left\{f\left(z_{i}\right)+\ln \left(1-p_{o}\right), f\left(n_{i}\right)+\ln p_{o}\right\} . \\
& \quad= \begin{cases}\ln \frac{1-p_{o}}{\sqrt{2 \pi \sigma_{i}^{2}}}-\frac{\left(z_{i}-d_{i}\right)^{2}}{\sigma_{i}^{2}}, & d_{i}\left(2 z_{i}-d_{i}\right) \geq \epsilon, \\
\ln \frac{p_{o}}{\sqrt{2 \pi \sigma_{i}^{2}}}-\frac{\left(z_{i}\right)^{2}}{\sigma_{i}^{2}}, & d_{i}\left(2 z_{i}-d_{i}\right)<\epsilon,\end{cases}
\end{aligned}
$$

where the threshold is $\epsilon=\sigma_{i}^{2} \ln \frac{p_{o}}{1-p_{o}}$.

For the case of $d_{i}\left(2 z_{i}-d_{i}\right) \geq \epsilon$, the second-order partial derivative is computed as

$$
\frac{\partial^{2} \ln p\left(z_{i} \mid \mathbf{x}\right)}{\partial \mathbf{x}^{2}}=\frac{1}{\sigma_{i}^{2}}\left[\begin{array}{cc}
J_{11}^{2} & J_{11} J_{21} \\
J_{21} J_{11} & J_{21}^{2}
\end{array}\right],
$$

with $J_{11}, J_{21}$ defined previously. For the other case, the partial derivative gives a zero matrix $\mathbf{0}$. This indicates no "information" is provided by faulty sensors for locating the target. Then the total FIM F can then be calculated, and the CRB is given by $\operatorname{cov}(\hat{\mathbf{x}}) \succeq \mathbf{F}^{-1}$.

\section{Simulation Results And Discussions}

Let us consider a $200 \times 200 \mathrm{~m}^{2}$ plane with one moving target, one transmitter and 6 receivers. The speeds of the target along the $x$ and $y$-axes are randomly drawn from the range $(0,3] \mathrm{m} / \mathrm{s}$. The positions of transceivers are assumed to be fixed, i.e. $\mathbf{x}_{t}=[0,0]^{\mathrm{T}}, \mathbf{x}_{1}=[80,10]^{\mathrm{T}}, \mathbf{x}_{2}=[10,80]^{\mathrm{T}}$, $\mathbf{x}_{3}=[130,20]^{\mathrm{T}}, \mathbf{x}_{4}=[20,130]^{\mathrm{T}}, \mathbf{x}_{5}=[180,30]^{\mathrm{T}}, \mathbf{x}_{6}=$ $[30,180]^{\mathrm{T}}$. A receiver is in the neighboring set $\mathcal{S}_{i}$ of receiver $i$, if and only if the two receivers are within the communication radius of $R=70 \mathrm{~m}$. For brevity, the measurement noise is set to $\sigma_{i}^{2}=2 m^{2}, \forall i \in \mathcal{M}$, unless otherwise specified. We consider 20 time slots and the number of message passing iterations during a single time slot is $L=10$.

Fig. 7 illustrates the cumulative distribution function (CDF) of target location error for the proposed algorithm and that of the state-of-the-art benchmark algorithms. The initialized uncertainties of the transceiver positions during the first time slot are set identically as $\vec{V}_{\mathbf{x}_{i, 0}}=\vec{V}_{\mathbf{x}_{t}^{0}}=25 \mathrm{~m}^{2}, \forall i \in \mathcal{M}$. At time slot $k$, the initial guesses of the transceivers and the target' locations for Taylor expansion are sampled from their prior distributions at the $k$ th time instant. The state transition noise $\boldsymbol{\Delta}_{s}$ has the covariance matrix $\mathbf{V}_{s}=\operatorname{diag}\left\{1 \mathrm{~m}^{2}, 1 \mathrm{~m}^{2}\right\}$. Observe from Fig. 7 that the TSE method [11] which neglects the uncertainties of the transceivers suffers from certain performance loss. Since the extended Kalman filtering (EKF) method regards the uncertainties as noise, the performance also degrades. As for the extension of the MOM-WLS estimator of [12], since the variance of the target position is not taken into consideration, the performance is adversely affected. The proposed factor graph based method outperforms the aforementioned algorithms and has a similar performance to the PF based method of [14], but significantly reduces the complexity.

The CDFs of target location of the proposed algorithm and of the TSE method in an asynchronous network are depicted in Fig. 8. It is assumed that the clock skew is drawn from a normal distribution $p(\alpha) \sim \mathcal{N}\left(1, V_{\alpha}^{0}\right)$, while the clock 


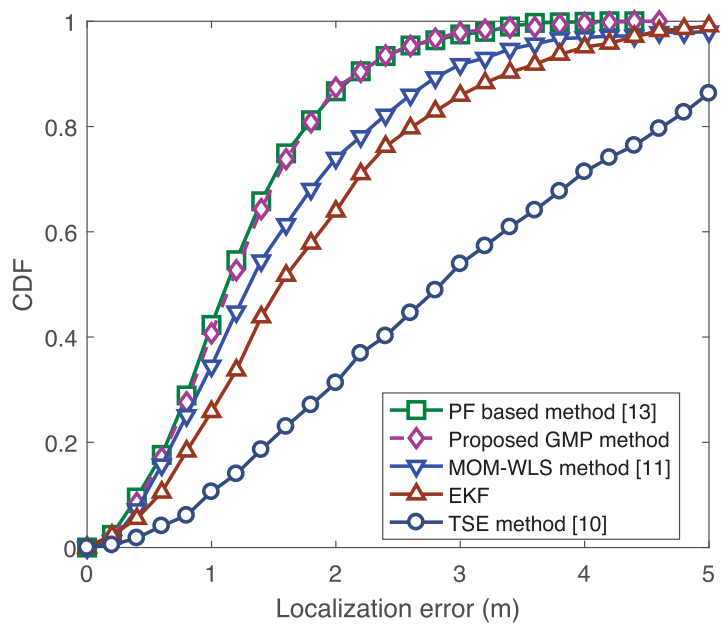

Fig. 7. Performance comparison to the state-of-the-art benchmark algorithms with uncertain transceiver positions.

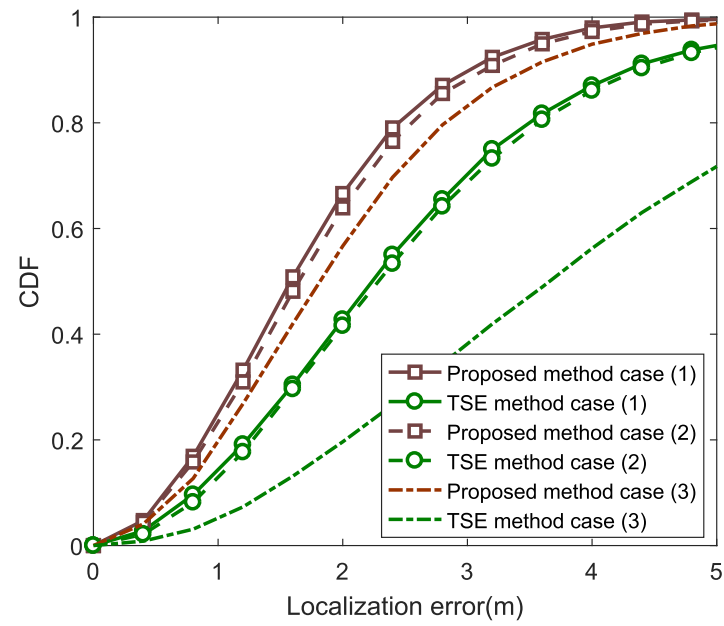

Fig. 8. Performance comparison of the proposed algorithm and the TSE method for different clock parameters.

offsets are drawn from a uniform distribution, or equivalently, from a Gaussian distribution having large variance of $p\left(\beta_{i}\right) \sim \mathcal{N}\left(0, \sigma_{\beta_{i}}^{2}\right), \forall i$. The variance of state transition noise is $V_{\beta}=10(n s)^{2}$. To evaluate the impact of clock parameters, three cases having varying a priori parameters are considered, i.e. (1) $V_{\alpha}^{0}=10^{-4}, \sigma_{\beta_{i}}=1 \mu s$; (2) $V_{\alpha}^{0}=10^{-8}, \sigma_{\beta_{i}}=1 \mu s$; (3) $V_{\alpha}^{0}=10^{-4}, \sigma_{\beta_{i}}=5 \mu s$. By comparing (1) and (3), we see that the performance gap between the proposed algorithm and the TSE method becomes much higher as the ignorance of clock information becomes severer. However, by comparing (1) and (2), it is noted that their performance is nearly the same, which means that $\alpha$ has only a modest impact on the localization result since its a priori distribution is accurate.

In Fig. 9, the root mean squared error (RMSE) of clock offsets versus the number of iterations is plotted. The performance of PF based method and of a regularized least square (RLS) based method are also shown as benchmarks. It is noted that all three algorithms converge after a few iterations. Due to the linearization of the range measurement, the proposed algorithm and the EKF method converge slower than the high-complexity PF based method. Moreover, after

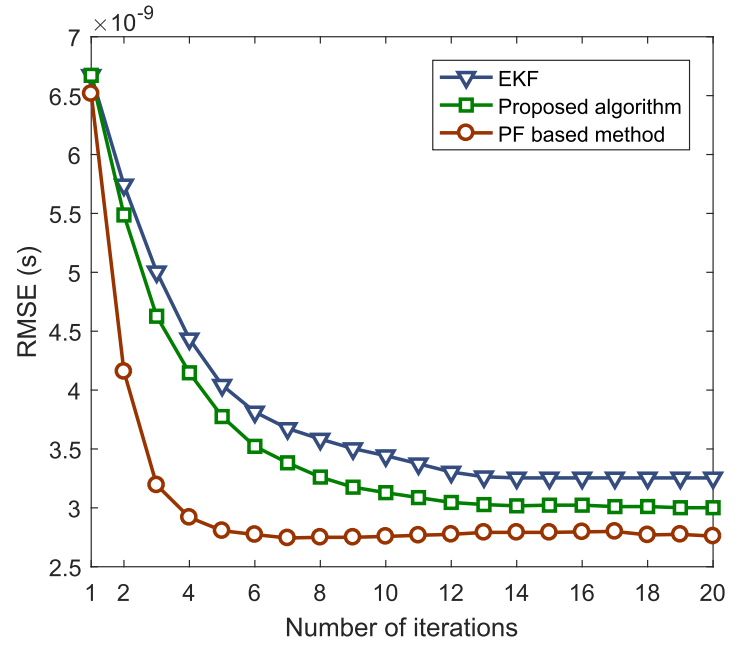

Fig. 9. RMSE of clock offsets versus the number of iterations.

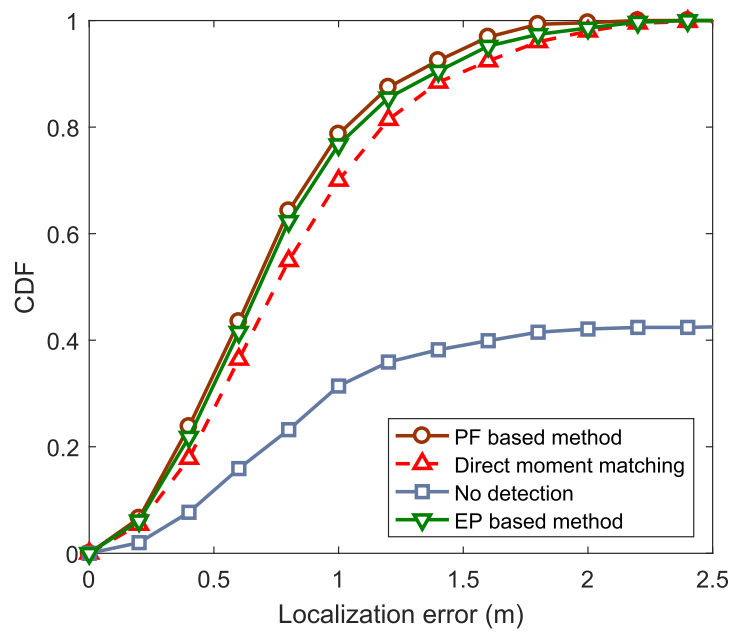

Fig. 10. CDF of target location of the proposed algorithm with the occupance probability $p_{o}=0.2$.

reaching convergence, the performance of the proposed algorithm approaches that of the PF based method and outperforms the EKF method.

Next, we will consider the robustness of the proposed algorithm in the presence of sensor detection failures. Observe in Fig. 10 that the CDF of target location in conjunction with the occupancy probability $p_{o}=0.2$ is plotted. When the faulty measurements observed at sensors are used without detection, the localization performance is seriously affected. The PF based method operating without Gaussian approximation has the best performance, albeit at the cost of a high complexity. We can further observe that using EP to approximate the discrete variable by a Gaussian variable has a better performance than matching the moments of its a priori distribution directly. This is because EP can exploit the information obtained from the measurements, or in other words, the extrinsic information.

Then in Fig. 11 the mean squared error (MSE) of target location is depicted for $p_{o} \in[0.1,0.9]$. The approximated CRB derived from the FIM (80) and the true CRB obtained using numerical methods are shown as benchmarks. It is observed that the approximated CRB is close to the true one. In the 


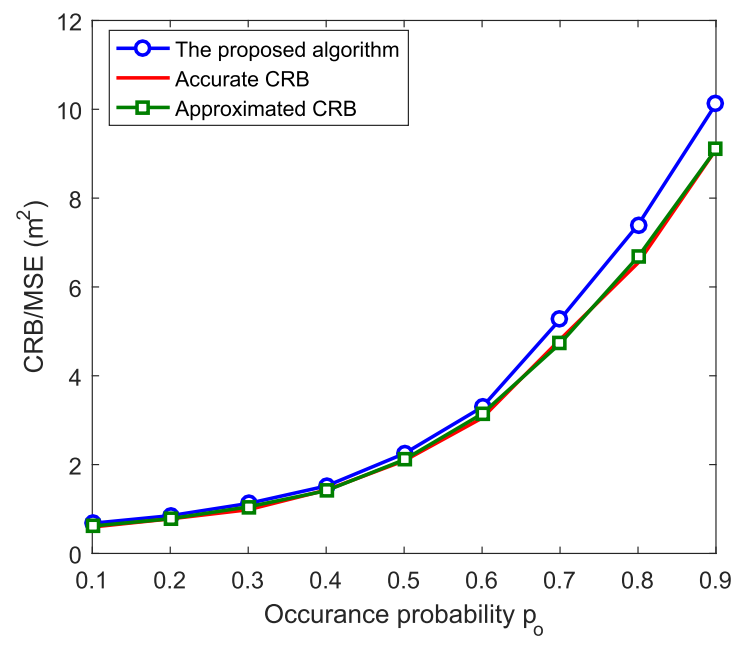

Fig. 11. MSE of the proposed algorithm and the derived CRB versus the occupance probability $p_{o}$.

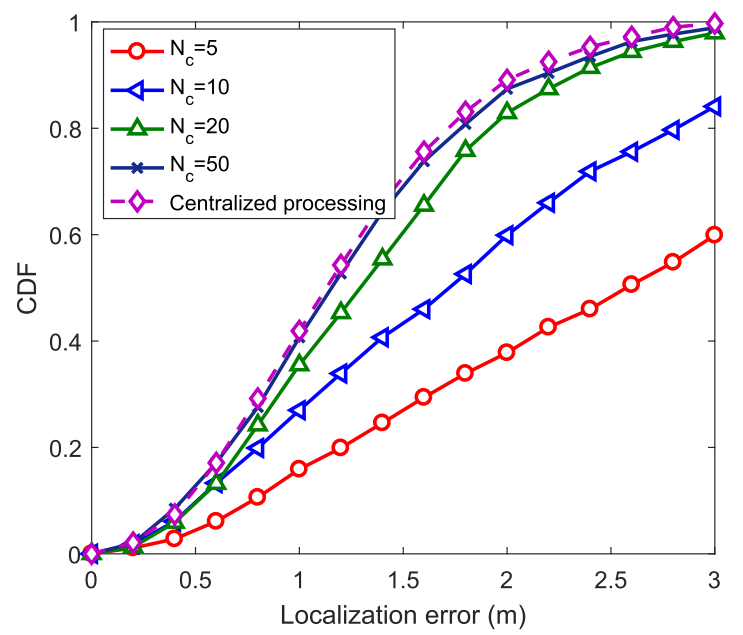

Fig. 12. Impact of the number of iterations $N_{c}$ for distributed processing.

region of small $p_{o}$, the proposed scheme is shown to be robust to link failures. When $p_{o}$ becomes larger, both the MSE of the proposed algorithm and the CRB degrade due to the reduced number of functioning sensors. In this situation, the Fisher information solely depends on $\mathbf{F}_{b}$ gleaned from the previous state.

To evaluate the proposed distributed processing method, we consider the scenario of Fig. 7. The receivers cooperate with their neighbors to reach consensus concerning the parameters corresponding to the target location. The CDFs of target location with different number of consensus processes are plotted in Fig. 12. We can see that the increase of $N_{c}$ helps improve the performance. However, the performance gain becomes marginal beyond a certain $N_{c}$. Bearing in mind that the communication overhead and energy cost are related to $N_{c}$, we can choose an appropriate value of $N_{c}$ to reduce the cost.

The MSEs of the target locations of all three circumstances versus the measurement noise are illustrated in Fig. 13, where the parameters are set as in Fig. 7, 8, 10. The MSEs of the distributed processing method with $N_{c}=20$ and the corresponding CRBs are also plotted. It can be seen that

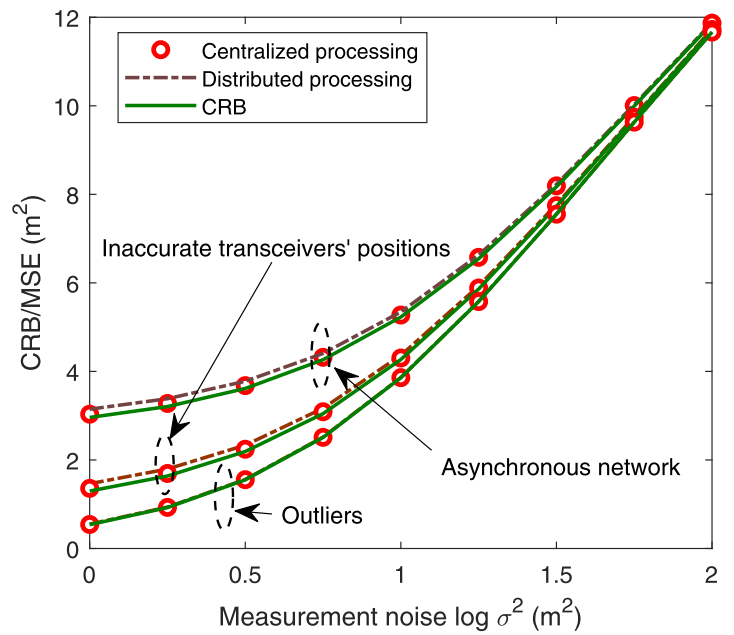

Fig. 13. Localization performance in different scenarios versus the measurement noise.

the proposed algorithm performs very close to the CRBs, which verifies the effeciency of the proposed centralized and distributed methods.

\section{CONCLUSION}

In this contribution, we provided a unified treatment of three major challenging issues, namely of the inaccurate positions of transceivers, of asynchronous network and of outliers in TOA based passive localization relying on a factor graph based framework and employed GMP techniques for solving them efficiently. We linearized the range measurement to obtain a linear state-space model for the proposed passive localization problems to reduce the complexity of PF based method. Considering that the fusion centers are unavailable in the energy constrained WSNs, we proposed distributed methods based on the consensus concept to determine the parameters distributively at each local receiver. Moreover, the Cramér-Rao bound was derived for characterizing the localization performance. Our simulation results showed that the performance of the proposed algorithm is close to that of the high-complexity PF based method and can approach the Cramér-Rao bound.

\section{APPENDIX A \\ Derivation of Variational Message PASSING IN GAUSSIAN FORM}

We first consider the calculation of message $\mu_{\delta \rightarrow x}$ based on SPA rules under multiplication constraint $\delta(z-x y)$ and Gaussian assumption of $\mu_{y \rightarrow \delta}(y) \propto \mathcal{N}\left(y, m_{y}, V_{y}\right)$,

$$
\begin{aligned}
\mu_{\delta \rightarrow x}(x) & =\int \delta(z-x y) \mu_{y \rightarrow \delta}(y) \mathrm{d} y \\
& \propto \exp \left(-\frac{\left(\frac{z}{x}-m_{y}\right)^{2}}{2 V_{y}}\right)=\exp \left(-\frac{m_{y}^{2}\left(x-\frac{z}{m_{y}}\right)^{2}}{2 x^{2} V_{y}}\right) .
\end{aligned}
$$

Note that the Equation (81) cannot be written in a form of Gaussian distribution, since the variable $x$ appears both in the numerator and in the denominator. Correspondingly, 
the message $\mu_{\delta \rightarrow y}(y)$ suffers from the same problem. In order to implement the low complexity Gaussian message passing, we resort the VMP concept of [40].

The message in VMP is obtained via expectation of the log likelihood function with respect to other variables, i.e.

$$
\mu_{f \rightarrow x}(x)=\exp \left[\mathbb{E}_{\mathbf{y}}(\ln f(x, \mathbf{y}) b(\mathbf{y}))\right]
$$

where $b(\mathbf{y}))$ is the belief of $\mathbf{y}$. Considering the multiplier node in Fig. 6, the observation model is $z_{i}^{k}=\phi_{i}^{k} r_{i}^{k}+n_{i}$. Hence the message from $₫$ to $\phi_{i}^{k}$ is calculated as,

$$
\begin{aligned}
& \mu_{\times \rightarrow \phi_{i}^{k}}\left(\phi_{i}^{k}\right) \\
& \propto C \cdot \exp \left(-\int \frac{\left(z_{i}^{k}-\phi_{i}^{k} r_{i}^{k}\right)^{2}}{2 \sigma_{i}^{2}} \exp \left(-\frac{\left(r_{i}^{k}-m_{r_{i}^{k}}\right)^{2}}{2 V_{r_{i}^{k}}}\right)\right) \\
& \quad \propto \exp \left(-\left(\phi_{i}^{k}\right)^{2} \frac{V_{r_{i}^{k}}+m_{r_{i}^{k}}^{2}}{\sigma^{2}}+2 \phi_{i}^{k} \frac{z_{i}^{k} m_{r_{i}^{k}}}{\sigma^{2}}\right) \\
& \propto \mathcal{N}\left(\phi_{i}^{k}, \overleftarrow{m}_{\phi_{i}^{k}}, \overleftarrow{V}_{\phi_{i}^{k}}\right)
\end{aligned}
$$

with $\overleftarrow{m}_{\phi_{i}^{k}}$ and $\overleftarrow{V}_{\phi_{i}^{k}}$ shown in (54) and (55). Finally, $\overleftarrow{m}_{r_{i}^{k}}, \overleftarrow{V}_{r_{i}^{k}}$ are determined in a similar way, which are also in Gaussian form.

\section{REFERENCES}

[1] I. F. Akyildiz, W. Su, Y. Sankarasubramaniam, and E. Cayirci, "A survey on sensor networks," IEEE Commun. Mag., vol. 40, no. 8, pp. 102-114, Aug. 2002.

[2] W. Dai, Y. Shen, and M. Z. Win, "Energy-efficient network navigation algorithms," IEEE J. Sel. Areas Commun., vol. 33, no. 7, pp. 1418-1430, Jul. 2015.

[3] H. Fu, S. Abeywickrama, L. Zhang, and C. Yuen, "Low-complexity portable passive drone surveillance via SDR-based signal processing," IEEE Commun. Mag., vol. 56, no. 4, pp. 112-118, Apr. 2018.

[4] Q. H. Spencer, B. D. Jeffs, M. A. Jensen, and A. L. Swindlehurst, "Modeling the statistical time and angle of arrival characteristics of an indoor multipath channel," IEEE J. Sel. Areas Commun., vol. 18, no. 3, pp. 347-360, Mar. 2000.

[5] E. Xu, Z. Ding, and S. Dasgupta, "Source localization in wireless sensor networks from signal time-of-arrival measurements," IEEE Trans. Signal Process., vol. 59, no. 6, pp. 2887-2897, Jun. 2011.

[6] J. Wang, Q. Gao, H. Wang, P. Cheng, and K. Xin, "Device-free localization with multidimensional wireless link information," IEEE Trans. Veh. Technol., vol. 64, no. 1, pp. 356-366, Jan. 2015.

[7] J. Wang, Q. Gao, H. Wang, Y. Yu, and M. Jin, "Time-of-flight-based radio tomography for device free localization," IEEE Trans. Wireless Commun., vol. 12, no. 5, pp. 2355-2365, May 2013.

[8] J. Wang, Q. Gao, Y. Yu, X. Zhang, and X. Feng, "Time and energy efficient TOF-based device-free wireless localization," IEEE Trans. Ind. Informat., vol. 12, no. 1, pp. 158-168, Feb. 2016.

[9] Y. Shen, W. Dai, and M. Z. Win, "Power optimization for network localization," IEEE/ACM Trans. Netw., vol. 22, no. 4, pp. 1337-1350, Aug. 2014.

[10] H. Wymeersch, J. Lien, and M. Z. Win, "Cooperative localization in wireless networks," Proc. IEEE, vol. 97, no. 2, pp. 427-450, Feb. 2009.

[11] J. Shen, A. F. Molisch, and J. Salmi, "Accurate passive location estimation using TOA measurements," IEEE Trans. Wireless Commun., vol. 11, no. 6, pp. 2182-2192, Jun. 2012.

[12] T. Qiao and H. Liu, "An improved method of moments estimator for TOA based localization," IEEE Commun. Lett., vol. 17, no. 7, pp. 1321-1324, Jul. 2013.

[13] J. Liu, Y. Zhang, and F. Zhao, "Robust distributed node localization with error management," in Proc. 7th ACM Int. Symp Mobile Ad Hoc Netw. Comput., 2006, pp. 250-261.

[14] N. Wu, W. Yuan, H. Wang, and J. Kuang, "TOA-based passive localization of multiple targets with inaccurate receivers based on belief propagation on factor graph," Digit. Signal Process., vol. 49, pp. 14-23, 2016.
[15] Y. Xiong, N. Wu, Y. Shen, and M. Z. Win, "Cooperative network synchronization: Asymptotic analysis," IEEE Trans. Signal Process., vol. 66, no. 3, pp. 757-772, Feb. 2018.

[16] J. Zheng and Y.-C. Wu, "Joint time synchronization and localization of an unknown node in wireless sensor networks," IEEE Trans. Signal Process., vol. 58, no. 3, pp. 1309-1320, Mar. 2010.

[17] A. Ahmad, E. Serpedin, H. Nounou, and M. Nounou, "Joint node localization and time-varying clock synchronization in wireless sensor networks," in Proc. IEEE Int. Conf. Acoust., Speech Signal Process., May 2013, pp. 5170-5174.

[18] W. Yuan, N. Wu, B. Etzlinger, H. Wang, and J. Kuang, "Cooperative joint localization and clock synchronization based on Gaussian message passing in asynchronous wireless networks," IEEE Trans. Veh. Technol. vol. 65, no. 9, pp. 7258-7273, Sep. 2016.

[19] Y. Zhou, C. Law, Y. Guan, and F. Chin, "Indoor elliptical localization based on asynchronous UWB range measurement," IEEE Trans. Instrum. Meas., vol. 60, no. 1, pp. 248-257, Jan. 2011.

[20] Y. Wang, S. Ma, and C. L. P. Chen, "TOA-based passive localization in quasi-synchronous networks," IEEE Commun. Lett., vol. 18, no. 4, pp. 592-595, Apr. 2014.

[21] W. Yuan, N. Wu, B. Etzlinger, Y. Li, C. Yan, and L. Hanzo, "Expectation-maximization-based passive localization relying on asynchronous receivers: Centralized versus distributed implementations," IEEE Trans. Commun., vol. 67, no. 1, pp. 668-681, Jan. 2019.

[22] V. J. Hodge and J. Austin, "A survey of outlier detection methodologies," Artif. Intell. Rev., vol. 22, no. 2, pp. 85-126, 2004.

[23] Y.-C. Chen and J.-C. Juang, "Outlier-detection-based indoor localization system for wireless sensor networks," Int. J. Navigat. Observ., vol. 2012, Feb. 2012, Art. no. 961785.

[24] L. Jian, Z. Yang, and Y. Liu, "Beyond triangle inequality: Sifting noisy and outlier distance measurements for localization," in Proc. IEEE INFOCOM, Mar. 2010, Art. no. 26.

[25] J. N. Tsitsiklis, "Problems in decentralized decision making and computation," DTIC, Fort Belvoir, VA, USA, Tech. Rep. LIDS-TH-1424, 1984.

[26] F. Meyer, E. Riegler, O. Hlinka, and F. Hlawatsch, "Simultaneous distributed sensor self-localization and target tracking using belief propagation and likelihood consensus," in Proc. 46th Asilomar Conf. Signals, Sys., Comput., Nov. 2012, pp. 1212-1216.

[27] O. Hlinka, F. Hlawatsch, and P. M. Djuric, "Distributed particle filtering in agent networks: A survey, classification, and comparison," IEEE Signal Process. Mag., vol. 30, no. 1, pp. 61-81, Jan. 2013.

[28] H.-A. Loeliger, J. Dauwels, J. Hu, S. Korl, L. Ping, and F. R. Kschischang, "The factor graph approach to model-based signal processing," Proc. IEEE, vol. 95, no. 6, pp. 1295-1322, Jun. 2007.

[29] J. Wang, Q. Gao, X. Zhang, H. Wang, and M. Jin, "Particle filter based device free localisation and tracking for large scale wireless sensor networks," Int. J. Sensor Net., vol. 19, nos. 3-4, pp. 194-203, 2015.

[30] L. Liu, C. Yuen, Y. L. Guan, Y. Li, and Y. Su, "Convergence analysis and assurance for Gaussian message passing iterative detector in massive MU-MIMO systems," IEEE Trans. Wireless Commun., vol. 15, no. 9, pp. 6487-6501, Sep. 2016

[31] W. Yuan, Q. Shi, N. Wu, Q. Guo, and X. Huang, "Gaussian message passing based passive localization in the presence of receiver detection failures," in Proc. IEEE 87th Veh. Technol. Conf., Jun. 2018, pp. 1-5.

[32] T. E. Fortmann, Y. Bar-Shalom, and M. Scheffe, "Sonar tracking of multiple targets using joint probabilistic data association," IEEE J. Ocean. Eng., vol. OE-8, no. 3, pp. 173-184, Jul. 1983.

[33] B. Ristic, B.-N. Vo, D. Clark, and B.-T. Vo, "A metric for performance evaluation of multi-target tracking algorithms," IEEE Trans. Signal Process., vol. 59, no. 7, pp. 3452-3457, Jul. 2011.

[34] J. Salmi and A. F. Molisch, "Propagation parameter estimation, modeling and measurements for ultrawideband MIMO radar," IEEE Trans. Antennas Propag., vol. 59, no. 11, pp. 4257-4267, Nov. 2011.

[35] F. R. Kschischang, B. J. Frey, and H.-A. Loeliger, "Factor graphs and the sum-product algorithm," IEEE Trans. Inf. Theory, vol. 47, no. 2, pp. 498-519, Feb. 2001.

[36] S. Kullback and R. A. Leibler, "On information and sufficiency," Annal. Math. Statist., vol. 22, no. 1, pp. 79-86, 1951.

[37] K. L. Noh, Q. M. Chaudhari, E. Serpedin, and B. W. Suter, "Novel clock phase offset and skew estimation using two-way timing message exchanges for wireless sensor networks," IEEE Trans. Commun., vol. 55, no. 4, pp. 766-777, Apr. 2007.

[38] A. Ahmad, D. Zennaro, E. Serpedin, and L. Vangelista, "A factor graph approach to clock offset estimation in wireless sensor networks," IEEE Trans. Inf. Theory, vol. 58, no. 7, pp. 4244-4260, Jul. 2012. 
[39] T. P. Minka, "Expectation propagation for approximate Bayesian inference," in Proc. Conf. Uncertainty Artif. Intell. Burlington, MA, USA: Morgan Kaufmann, 2001, pp. 362-369.

[40] J. M. Winn and C. M. Bishop, "Variational message passing," J. Mach. Learn. Res., vol. 6, pp. 661-694, Apr. 2005.

[41] O. Hlinka, O. Slučiak, F. Hlawatsch, P. M. Djurić, and M. Rupp, "Likelihood consensus and its application to distributed particle filtering," IEEE Trans. Signal Process., vol. 60, no. 8, pp. 4334-4349, Apr. 2012.

[42] T. C. Aysal, M. E. Yildiz, A. D. Sarwate, and A. Scaglione, "Broadcast gossip algorithms for consensus," IEEE Trans. Signal Process., vol. 57, no. 7, pp. 2748-2761, Jul. 2009

[43] H. V. Poor, An Introduction to Signal Detection and Estimation. Berlin, Germany: Springer, 2013

[44] T. Wang, Y. Shen, A. Conti, and M. Z. Win, "Network navigation with scheduling: Error evolution," IEEE Trans. Inf. Theory, vol. 63, no. 11, pp. 7509-7534, Nov. 2017

[45] Y. Han et al., "Performance limits and geometric properties of array localization," IEEE Trans. Inf. Theory, vol. 62, no. 2, pp. 1054-1075, Feb. 2016

[46] J. Stewart, Calculus. Boston, MA, USA: Cengage Learning, 2011.

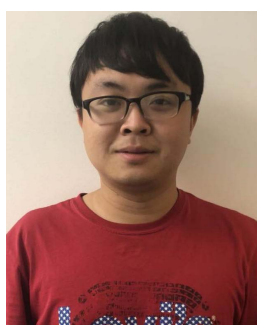

Weijie Yuan (S'15) received the Ph.D. degree from the Beijing Institute of Technology, China, and the Ph.D. degree from the University of Technology Sydney, Australia, in 2019. He is currently a Research Associate with the School of Electrical Engineering and Telecommunications, University of New South Wales, Sydney, Australia. He has served as a Research Assistant with the University of Sydney, a Visiting Associate Fellow with the University of Wollongong, and a Visiting Fellow with the University of Southampton, from 2017 to 2019 In 2016, he was a Visiting Ph.D. Student with the Institute of Telecommunications, Vienna University of Technology, Austria. His research interest includes statistical inference on graphical models. He has served as a TPC member for several conferences.

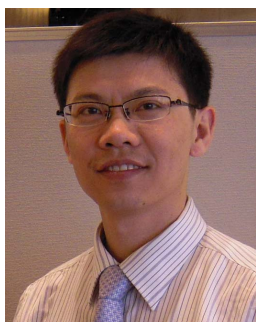

Nan Wu (M'11) received the B.S., M.S., and Ph.D. degrees from the Beijing Institute of Technology (BIT), Beijing, China, in 2003, 2005, and 2011, respectively. From 2008 to 2009, he was a Visiting $\mathrm{Ph} . \mathrm{D}$. Student with the Department of Electrical Engineering, Pennsylvania State University, USA. $\mathrm{He}$ is currently a Professor with the School of Information and Electronics, BIT. His research interests include signal processing in wireless communication networks. He serves as an Editorial Board Member for IEEE ACCESS, International Journal of Electronics and Communications, KSII Transactions on Internet and Information Systems, and IEICE Transactions on Communications. He was a recipient of the National Excellent Doctoral Dissertation Award from the MOE of China in 2013

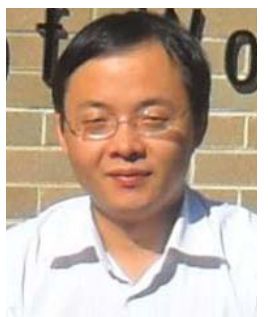

Qinghua Guo (S'07-M'08-SM'18) received the B.E. degree in electronic engineering and the M.E. degree in signal and information processing from Xidian University, Xi' an, China, in 2001 and 2004 respectively, and the Ph.D. degree in electronic engineering from the City University of Hong Kong, Hong Kong, in 2008. He is currently an Associate Professor with the School of Electrical, Computer and Telecommunications Engineering, University of Wollongong, Wollongong, NSW, Australia, and an Adjunct Associate Professor with the School of Engineering, The University of Western Australia, Perth, WA, Australia. His research interests include signal processing and telecommunications. He was a recipient of the Australian Research Council's Inaugural Discovery Early Career Researcher Award in 2012

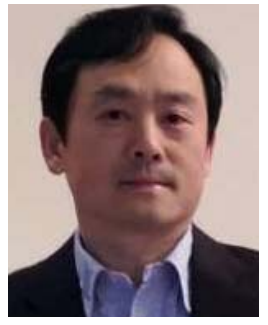

Xiaojing Huang (M'99-SM'11) received the B.Eng., M.Eng., and Ph.D. degrees in electronic engineering from Shanghai Jiao Tong University, Shanghai, China, in 1983, 1986, and 1989, respectively. He was a Principal Research Engineer with the Motorola Australian Research Center, Botany, NSW, Australia, from 1998 to 2003, and an Associate Professor with the University of Wollongong, Wollongong, NSW, Australia, from 2004 to 2008. He has been a Principal Research Scientist with the Commonwealth Scientific and Industrial Research Organisation (CSIRO), Sydney, NSW, Australia, and the Project Leader of the CSIRO microwave and mm-wave Backhaul projects since 2009. He is currently a Professor of information and communications technology with the School of Electrical and Data Engineering and the Program Leader for Mobile Sensing and Communications with the Global Big Data Technologies Center, University of Technology Sydney (UTS), Sydney. With over 30 years of combined industrial, academic, and scientific research experience, he has authored over 300 book chapters, refereed journals and conference papers, and major commercial research reports. He has filed 31 patents. He was a recipient of the CSIRO Chairman's Medal and the Australian Engineering Innovation Award in 2012 for exceptional research achievements in multigigabit wireless communications.

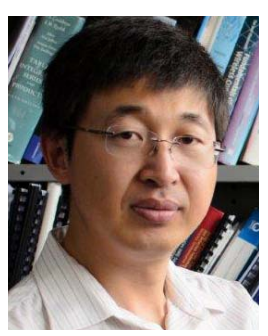

Yonghui Li (M'04-SM'09-F'19) received the Ph.D degree from the Beijing University of Aeronautics and Astronautics in 2002.

From 1999 to 2003, he was affiliated with Linkair Communication Inc., where he was the Project Manager responsible for the design of physical layer solutions for the LAS-CDMA system. Since 2003, he has been with the Centre of Excellence in Telecommunications, University of Sydney, Australia. He is currently a Professor with the School of Electrical and Information Engineering, University of Sydney. He holds a number of patents granted and pending in these fields. His current research interests are in the areas of wireless communications, with a particular focus on MIMO, millimeter wave communications, machine to machine communications, coding techniques, and cooperative communications. He was a recipient of the Australian Queen Elizabeth II Fellowship in 2008 and the Australian Future Fellowship in 2012. He also served as a Guest Editor for several special issues of IEEE journals, such as IEEE Journal on SELeCted AREAS in Communications Special Issue on Millimeter Wave Communications. He was a recipient of the Best Paper Award from IEEE International Conference on Communications (ICC) 2014, IEEE PIMRC 2017, and IEEE Wireless Days Conferences (WD) 2014. He is currently an Editor of IEEE TRANSACTIONS ON COMMUNICATIONS and IEEE TRANSACTIONS ON VEHICULAR TECHNOLOGY.

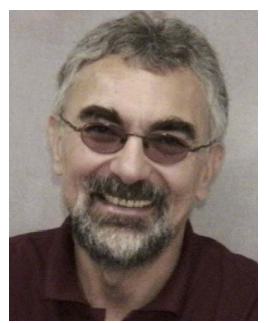

Lajos Hanzo (M'91-SM'92-F'04) received the D.Sc. degree in electronics in 1976 and the Ph.D. degree in 1983. He received an Honorary Ph.D. degree from the Technical University of Budapest and the University of Edinburgh in 2009 and 2015, respectively. In 2016, he was admitted to the Hungarian Academy of Science. During his 40-year career in telecommunications, he has held various research and academic posts in Hungary, Germany, and U.K. Since 1986, he has been with the School of Electronics and Computer Science, University of Southampton, U.K., where he holds the chair of telecommunications. He has successfully supervised $111 \mathrm{Ph} . \mathrm{D}$. students. He has co-authored $18 \mathrm{John}$ Wiley/IEEE Press books on mobile radio communications totaling in excess of 10000 pages. He has published 1756 research contributions at the IEEE Xplore. He has acted both as the TPC and the General Chair of IEEE conferences. He has presented keynote lectures and has been awarded a number of distinctions. He is currently the Director of a 60 -strong academic research team, working on a range of research projects in the field of wireless multimedia communications sponsored by industry, the Engineering and Physical Sciences Research Council (EPSRC), U.K. He is a fellow of the Royal Academy of Engineering, the IET, and the EURASIP. He has received the European Research Council's Advanced Fellow Grant and the Royal Society's Wolfson Research Merit Award. He is an enthusiastic supporter of industrial and academic liaison, and offers a range of industrial courses. He is also a Governor of the IEEE ComSoc and VTS. From 2008 to 2012, he was the Editor-in-Chief of IEEE Press and the Chair Professor with Tsinghua University, Beijing. 